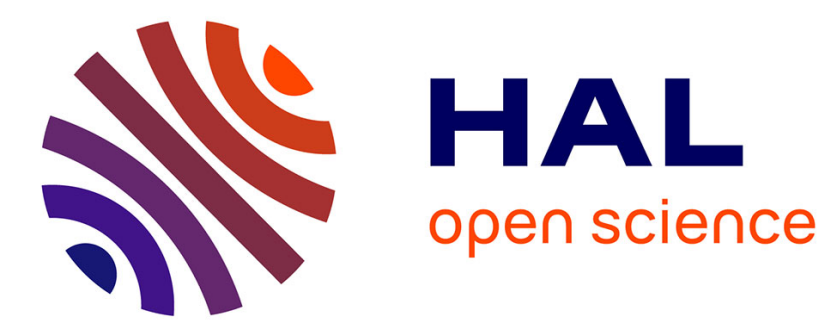

\title{
Ressources économiques des femmes et travail domestique des conjoints: quels effets pour quelles tâches?
}

Simon Bittmann

\section{- To cite this version:}

Simon Bittmann. Ressources économiques des femmes et travail domestique des conjoints: quels effets pour quelles tâches?. Economie et Statistique / Economics and Statistics, 2015, 478-479-480, pp.305 - 338. 10.3406/estat.2015.10566 . hal-01309219

HAL Id: hal-01309219

https://hal-sciencespo.archives-ouvertes.fr/hal-01309219

Submitted on 29 Apr 2016

HAL is a multi-disciplinary open access archive for the deposit and dissemination of scientific research documents, whether they are published or not. The documents may come from teaching and research institutions in France or abroad, or from public or private research centers.
L'archive ouverte pluridisciplinaire HAL, est destinée au dépôt et à la diffusion de documents scientifiques de niveau recherche, publiés ou non, émanant des établissements d'enseignement et de recherche français ou étrangers, des laboratoires publics ou privés. 


\title{
Ressources économiques des femmes et travail domestique des conjoints : quels effets pour quelles tâches?
}

\author{
Simon Bittmann *
}

\begin{abstract}
Cet article étudie l'impact des revenus du travail sur le temps de travail domestique des femmes françaises au sein de 1674 couples bi-actifs occupés à temps plein, à partir de l'enquête Emploi du temps 2009-2010. Nous testons deux théories visant à interpréter le rapport entre les ressources économiques des conjoints et le temps alloué aux tâches domestiques. L'approche « absolue » explique la diminution du temps que les femmes y consacrent par l'augmentation de leur salaire, indépendamment de celui du conjoint, alors que l'approche « relative » met en avant le rôle des salaires relatifs comme mesure du pouvoir de négociation des femmes au sein du couple. Nous montrons que l'effet non linéaire observé pour le salaire relatif, interprété en termes de «performance de genre » dans la littérature, est dû à une mauvaise caractérisation de la relation entre salaire et temps de travail domestique. Celle-ci est très fortement non linéaire pour les femmes, indépendamment de leur pouvoir de négociation : à mesure que le salaire augmente, l'élasticité de leur temps de travail domestique au salaire se tasse. Il semble ainsi y avoir un niveau minimal de tâches incompressible pour les femmes, et cette diminution s'opère avant tout par une coupe dans les tâches ménagères et la cuisine ; le temps consacré aux enfants est insensible aux variations de revenus. La « relève » des hommes n'est que très partielle, et ne présente pas de forme non linéaire. De même, le recours à des services extérieurs explique très peu cette diminution ; il semble que les femmes adaptent avant tout leurs « attentes » par des modifications du volume global de production domestique. Ceci entraîne des changements du volume relatif de tâches effectué : une augmentation du poids relatif de la cuisine par rapport au ménage, et de « s'occuper » des enfants par rapport aux autres tâches.
\end{abstract}

Les jugements et opinions exprimés par les auteurs n'engagent qu'eux mêmes, et non les institutions auxquelles ils appartiennent, ni a fortiori l'Insee.
Codes JEL : D13, Z1.

Mots clés : travail domestique, salaire des femmes, genre. 
$\mathbf{C}$ et article vise à comprendre l'effet des ressources économiques des femmes sur la production domestique en France, cette question ayant donné lieu depuis une vingtaine d'années à de vifs débats internationaux en sociologie économique (England et Folbre, 2005 ; England, 2011; Sullivan, 2011 ; Gupta, 2006 et 2007). L'interrogation principale porte sur l'impact de l'amélioration de la situation économique des femmes sur la quantité de travail et le type de tâches domestiques qu'elles effectuent. Les femmes ont vu leur position sur le marché du travail s'améliorer et ceci a eu un impact négatif sur la quantité de temps qu'elles allouent aux tâches domestiques (Gershuny, 2003 ; Ponthieux et Schreiber, 2006 ; Rizavi et Sofer, 2008 ; Coltrane, 2000). Mais depuis les travaux fondateurs de Hochschild et Machung (1989), on sait que cette diminution ne se déroule pas sans frictions: les femmes effectuent encore à ce jour une part plus importante des tâches domestiques (cf. tableau 1), même au sein des couples bi-actifs occupés où les deux membres ont des niveaux de revenus comparables (Evertsson

\section{Tableau 1}

\section{Statistiques descriptives}

\begin{tabular}{|c|c|c|c|}
\hline Variable & $\begin{array}{l}\text { Femme } \\
(1674)\end{array}$ & $\begin{array}{l}\text { Homme } \\
(1674)\end{array}$ & $\begin{array}{l}\text { Différence } \\
\quad(\%)\end{array}$ \\
\hline Âge & $\begin{array}{l}42,46 \\
(0,20)\end{array}$ & $\begin{array}{l}40,56 \\
(0,20)\end{array}$ & \\
\hline \multicolumn{4}{|l|}{ Temps quotidien alloué au travail domestique (minutes) } \\
\hline Volume global & $\begin{array}{r}175,36 \\
(3,21)\end{array}$ & $\begin{array}{l}77,11 \\
(2,20)\end{array}$ & 124,80 \\
\hline Faire la cuisine & $\begin{array}{l}48,39 \\
(1,18)\end{array}$ & $\begin{array}{l}19,12 \\
(0,73)\end{array}$ & 153,00 \\
\hline Faire le ménage & $\begin{array}{l}58,42 \\
(1,59)\end{array}$ & $\begin{array}{l}23,01 \\
(1,00)\end{array}$ & 153,80 \\
\hline S'occuper du linge & $\begin{array}{l}20,78 \\
(0,95)\end{array}$ & $\begin{array}{r}1,36 \\
(0,21)\end{array}$ & 1427,90 \\
\hline S'occuper des enfants & $\begin{array}{l}39,52 \\
(1,74)\end{array}$ & $\begin{array}{l}18,51 \\
(1,10)\end{array}$ & 113,50 \\
\hline Répartition du travail domestique, niveau agrégé & 0,69 & 0,31 & 124,90 \\
\hline Temps quotidien alloué au travail salarié (minutes) & $\begin{array}{r}252,32 \\
(5,73)\end{array}$ & $\begin{array}{r}315,07 \\
(6,07)\end{array}$ & $-24,86$ \\
\hline Salaire mensuel & $\begin{array}{r}1649,18 \\
(19,96)\end{array}$ & $\begin{array}{r}2112,74 \\
(32,84)\end{array}$ & $-28,10$ \\
\hline Pourcentage d'individus ayant un salaire supérieur ou égal à celui de leur conjoint & 36 & 73,1 & 50,75 \\
\hline $\begin{array}{l}\text { Salaire des individus touchant un salaire supérieur } \\
\text { ou égal à celui de leur conjoint }\end{array}$ & $\begin{array}{r}2052,04 \\
(37,13)\end{array}$ & $\begin{array}{r}2305,13 \\
(42,1)\end{array}$ & $-10,98$ \\
\hline $\begin{array}{l}\text { Écart moyen avec le salaire du conjoint pour les individus touchant un salaire supérieur } \\
\text { à celui du conjoint }\end{array}$ & $\begin{array}{l}431,80 \\
(24,47)\end{array}$ & $\begin{array}{l}841,55 \\
(37,13)\end{array}$ & 49,69 \\
\hline \multicolumn{4}{|l|}{ Nombre d'enfants : } \\
\hline Âge $<3$ & $\begin{array}{r}0,13 \\
(0,005)\end{array}$ & - & - \\
\hline $3 \leq$ âge $<6$ & $\begin{array}{r}0,21 \\
(0,008)\end{array}$ & - & - \\
\hline $6 \leq$ âge $\leq 18$ & 0,72 & - & - \\
\hline Âge $\leq 18$ & $\begin{array}{r}1,06 \\
(0,018)\end{array}$ & - & - \\
\hline \multicolumn{4}{|l|}{ Le ménage a eu recours à des services extérieurs : } \\
\hline Pressing & 37,40 & - & - \\
\hline Aide rémunérée pour le ménage & 11,35 & - & - \\
\hline Commande et livraison de plats préparés & 21,98 & - & - \\
\hline Système de garde rémunérée des enfants & 29,84 & - & - \\
\hline
\end{tabular}

Lecture: les écarts-types sont fournis quand cela est nécessaire. La part agrégée est calculée sur la totalité de l'échantillon alors que les parts individuelles sont calculées par ménage. Nous ne considérons que les couples en cohabitation, ainsi le nombre d'enfants est le même pour les hommes et les femmes. De la même manière, le recours aux services extérieurs indique le pourcentage de ménages ayant indiqué avoir eu recours à ce types de solution au moins une fois durant la semaine de l'enquête.

Champ : couples hétérosexuels bi-actifs occupés, exerçant une activité salariée à temps complet, en situation de cohabitation. Source : enquête Emploi du Temps 2009-2010, Insee. 
et Nermo, 2007). Il y a une dizaine d'années, Ponthieux et Schreiber (2006) ont mis en évidence l'impact « dérisoire » du pouvoir économique des femmes sur la répartition du travail domestique au sein des couples français. Un objectif de cet article sera de développer et de tester plus systématiquement ce résultat à partir des données issues de l'enquête Emploi du temps 2009-2010. Il s'agira dans une première partie de décrire ces résistances, cette inélasticité des tâches aux améliorations du niveau de revenu des femmes, puis dans une deuxième partie de décrire comment s'opère cette diminution, par quelles tâches spécifiques l'observe-t-on et si celle-ci est compensée par une relève des hommes, un recours à des services extérieurs marchands ou si elle entraine simplement une diminution du temps global consacré aux tâches domestiques.

De nombreux travaux issus de la sociologie et de l'économie permettent de comprendre en quoi la réduction des inégalités économiques au sein des couples a pu modifier le temps consacré aux tâches domestiques par les deux conjoints. Les travaux qui s'intéressent à l'impact direct du salaire de la femme sur la quantité de travail domestique qu'elle effectue adoptent une perspective dite " absolue $»^{1}$, alors que les approches dites "relatives" insistent sur les ressources relatives des deux membres du ménage. Ce ne serait pas le niveau de salaire en lui-même mais une mesure du pouvoir de négociation de l'homme et de la femme qui permettrait de comprendre la répartition des tâches domestiques au sein des ménages français.

Ces deux approches ont souvent mis en avant une relation linéaire (négative) ${ }^{2}$ entre ressources économiques et quantité de travail domestique des membres du ménage, une prédiction invalidée par des études empiriques portant sur de nombreux pays ${ }^{3}$. Brines (1994) fut la première à identifier empiriquement une non-linéarité dans la relation entre ressources économiques et temps de travail domestique. L'article s'inscrit dans une approche pleinement « relative»; l'auteure utilise une mesure de la « dépendance économique » des conjoints, et met en évidence une non-linéarité extrême puisqu'elle est non-monotone dans le cas des hommes : au delà d'un certain niveau de salaire, elle observe un retournement de l'effet des ressources relatives d'un conjoint sur son temps de travail domestique. Si l'homme gagne en particulier moins que sa femme, il peut avoir tendance à prendre à sa charge encore moins de travail domestique, en compensation de cette déviance, normative ou identitaire, vis-à-vis de la division traditionnelle du travail au sein du ménage. Cette compensation a été présentée comme une des modalités d'expression de ce que Goffman (1979) nomme la "performance de genre»: les individus exprimeraient le coût psychologique ou social à s'écarter de la norme par une démonstration " compensatoire » de leur identité de genre et des attentes qui lui sont socialement rattachées. Il existerait alors un effet de second ordre, négatif pour l'homme (Brines, 1994) et positif pour la femme (Greenstein, 2000 ; Bertrand et al., 2015), qui expliquerait la stagnation des inégalités en termes de travail domestique, et ce malgré la diminution progressive des inégalités économiques. C'est une version extrême de ce mécanisme qui a été mise en avant par Brines (1994), puisque dans son article, celui-ci mène à un retournement de la courbe estimée, et nous qualifierons ce cas de «neutralisation de la déviance de genre $»^{4}$ (hypothèse « NDG ») .

La présence éventuelle de cet effet social spécifique de compensation, qui viendrait faire contrepoids aux logiques purement économiques, a été extrêmement débattue ces vingt dernières années au niveau international. Or cette discussion recoupe selon nous trois débats qu'il s'agit de différencier: le premier porte sur la manière de considérer les ressources économiques des conjoints, entre approches " absolue » et « relative ", le second concerne la forme fonctionnelle de la relation identifiée, linéaire ou non linéaire, et le troisième porte, le cas échéant, sur la monotonie de la courbe non linéaire identifiée. Nous résumons ces différents débats dans la figure $\mathrm{I}:$ la thèse de la «NDG» ne concerne qu'un cas limite où l'on observe un renversement de la courbe du travail domestique en fonction des ressources

\footnotetext{
1. Le terme qui apparaît dans les articles les plus récents est celui d' " autonomiste ", voir Gupta (2007) ou Killewald et Gough (2010). Nous avons choisi de recourir au terme "absolu », afin d'éviter toute confusion quant au fait que ce terme ne se rapporte pas aux modalités des choix effectués par les conjoints dans le couple, mais simplement au statut donné aux salaires des conjoints, considérés ici indépendamment l'un de l'autre.

2. Ces approches n'impliquent pas nécessairement une relation linéaire entre ressources économiques et temps de travail domestique, mais les travaux empiriques qui s'en revendiquent ont souvent utilisé une telle caractérisation dans leurs estimations.

3. Voir Akerlof et Kranton (2000) et Bertrand et al. (2015) pour les États Unis, Bittman et al. (2003) pour l'Australie, Bloemen et Stancanelli (2008) pour l'Italie, Hook (2010) pour une comparaison internationale et Sullivan (2011) pour une synthèse de ces différents travaux.

4. "Gender deviance neutralisation ", à la suite notamment de Bittman et al. (2003) et de Lyonette et Crompton (2014).

5. Il faut également noter que Brines inclut les chômeurs dans son échantillon, problème sur lequel nous reviendrons.
} 
Encadré

\section{APPROCHES « ABSOLUES » ET « RELATIVES »}

Les débats empiriques au sein desquels s'inscrit cet article s'appuient sur des fondements théoriques issus de la sociologie et de l'économie qu'il s'agit de présenter brièvement. Nous regroupons ces travaux non pas par discipline, mais sur la base des prédictions empiriques qu'ils mettent en avant, entre approches « absolues » et « relatives ».

\section{Les approches « absolues »}

Les modèles dits «unitaires " (Becker, 1963) sont les premiers à avoir insisté sur le salaire autonome de chaque conjoint comme variable principale dans le processus de décision du ménage. Gronau (1977) fut le premier à étendre le schéma beckerien du coût d'opportunité (Becker, 1963 et 2009) à la production du travail domestique, et, selon ce modèle, les choix du ménage peuvent être représentés par une fonction d'utilité unique qui permettra de comprendre la répartition du travail de marché et du travail domestique. Le salaire est simplement compris comme un coût d'opportunité qui guide les choix de participer ou non aux tâches domestiques, et ce modèle prédit une corrélation négative entre salaire et temps de travail domestique : à mesure que les revenus du travail augmentent, le coût d'opportunité de la consommation de "commodités " nécessitant relativement plus de temps que de biens augmente, en particulier le travail domestique, et leur consommation doit décroître. L'équilibre est caractérisé par une égalisation des productivités marginales du travail domestique et du travail salarié.

Le travail de Solberg et Wong (1992) a permis d'affiner ce modèle en introduisant un ménage à deux individus présentant des "productivités " domestiques différentes, tout en conservant l'idée que ce qui importe sont les revenus individuels des conjoints, qui sont mis en balance avec leur " profil » technologique. Les femmes étant supposées faire preuve de la productivité domestique la plus forte, ce sont elles qui font le plus de travail domestique ; aucun élément de décision collective au niveau du ménage ne joue dans ce modèle.

Une autre manière d'insister sur l'autonomie des conjoints consiste à interpréter le travail domestique comme une compensation fournie par les femmes en temps de travail non-rémunéré pour les apports économiques des hommes. Ces théories insistent sur la notion de "dépendance économique " des femmes (Delphy et Leonard, 1992 ; Delphy, 2001 ; Acker, 1988 ; Brines, 1994) : la femme prend à sa charge les tâches domestiques en échange du soutien économique garanti par son mari. Cette vision s'appuie davantage sur une lecture historique liée à l'émergence du modèle du breadwinner à l'époque industrielle (Horrell et Humphries, 1995 et 1996) et à la spécialisation des conjoints dans des sphères différentes de la production économique (marchande pour l'hommme, domestique et gratuite pour la femme). Selon cette approche, une augmentation des revenus des femmes devrait diminuer leur dépendance et ainsi la quantité de travail domestique qu'elles fournissent en échange de cela. En dehors de ces mécanismes de causalité spécifiques, l'approche appelée " autonomiste » dans la littérature américaine (Killewald et Gough, 2010) regroupe l'ensemble des mécanismes de causalité qui peuvent mettre en évidence un lien entre le salaire individuel de chaque conjoint et la quantité de temps alloué aux tâches domestiques. Empiriquement, cette piste a été principalement explorée par Gupta (2006 et 2007), Gupta et Ash (2008) et reprise par Sullivan (2011). Ces auteurs mettent en avant le pouvoir explicatif des ressources absolues, en particulier du salaire des femmes, pour comprendre l'évolution de la répartition du travail domestique au sein des ménages.

\section{Les approches « relatives »}

Les premiers articles issus de l'approche "relative " ont cherché à contester les modèles " unitaires " en ouvrant la «boîte noire " des décisions intra-ménage. Ces travaux s'appuient sur la théorie des jeux pour tenter de comprendre la répartition du travail domestique en fonction d'un processus de décision intra ménage. Dans ces modèles (Manser et Brown, 1980 ; McElroy et Horney, 1981 ; Chiappori, 1992 ; Chiappori et al., 2002), le ménage est caractérisé par deux fonctions d'utilité différentes pour l'homme et la femme, ainsi qu'un processus de décision collective déterminé par un algorithme de négociation. Pour cet algorithme, McElroy et Horney (1981) spécifient un processus de négociation de Nash, tandis que Chiappori (1992) part d'une critère d'efficacité de Pareto et que Manser et Brown (1980) utilisent un algorithme de Kalai-Smorodinsky. Le pouvoir de négociation de chaque membre du ménage y est déterminé par des " points de menace ", à savoir le niveau d'utilité obtenu par chaque individu si l'équilibre n'est pas atteint: si l'homme et la femme décident de se séparer, quelles seront les ressources économiques de chacun en situation de célibat? Les décisions au niveau du ménage sont donc le fruit d'un jeu coopératif où les ressources disponibles de chacun, en particulier le salaire, déterminent leur pouvoir de négociation. Ces modèles cherchaient avant tout à comprendre la formation des ménages, et la dynamique conjugale (célibat, mariage, divorce) en fonction de variables économiques mais Apps et Rees (1997) ont élargi ce schéma aux décisions relevant de la production de travail domestique (voir également Chiappori, 1997). Cette approche incite à prendre en compte une mesure du salaire relatif de chaque époux (Bertrand et al., 2015) : ceci est dû à l'apparition d'un rapport de salaire dans les conditions de première ordre des modèles introduisant une fonction de production domestique. Cette raison " structurelle " a justifié empiriquement l'introduction d'une mesure distributive ou relative des ressources des conjoints. En toute rigueur, ces modèles s'appuient sur une mesure relative des salaires " potentiels ", ce qui rend délicate l'évaluation empirique de leurs prédictions. Ce salaire « potentiel » est mesuré après la prise en considération de l'interaction entre les choix d'allocations du temps et les salaires futurs, par l'investissement en capital humain. D'autres approches (Brines, 1994 ; Greenstein, 2000 ; Bittman et al., 2003 ; Bertrand et al., 2015) se sont intéressées plus directement aux ressources relatives effectives (et non " potentielles ») pour mettre en avant les jeux de négociations ou d'échange qui pouvaient avoir lieu au sein des couples. Selon ces auteurs, le mariage représente une série de contrats informels qui déterminent la "règle de partage " (sharing rule) des différentes tâches, en particulier celles ayant trait au travail domestique au sein du ménage (voir Thibout, 
Encadré (suite)

2014, chapitre 2, pour une étude empirique sur la sharing rule au sein des couples français). Or ce sont les ressources économiques relatives qui permettent aux époux en devenir d'influencer la loi de répartition des tâches en leur faveur.

On peut constater que dans ces modèles, l'amélioration de la situation économique des femmes vis-à-vis de celle de leur conjoint devrait tendre à une égalisation progressive de la répartition du travail domestique : qu'on interprète cela comme une modification des avantages comparatifs, une augmentation du pouvoir de négociation des femmes, ou une réduction de la dépendance économique vis-à-vis du mari, on devrait observer un effet linéaire fort et continu des ressources relatives des femmes.

\section{Identité et performance de genre, l'effet non-linéaire des ressources relatives}

Lundberg et Pollak $(1993,1994)$ ont tenté de caractériser différemment les "points de menace " mentionnés précédemment: au sein d'un ménage marié, un équilibre non coopératif peut émerger lors du processus de négociation qui ne mène pas nécessairement au divorce mais à ce que les auteurs appellent un équilibre de " sphères séparées ". L'échec de la coopération aboutit à une situation où l'homme et la femme se conforment à une répartition traditionnelle des tâches : spécialisation relative dans la production marchande pour l'homme et dans la production domestique pour la femme. En des termes empruntés à Hirschman (1970), on pourrait dire que le choix d'، exit » caractérisé par le divorce ne serait pas la seule option extérieure disponibles aux époux, ceux-ci peuvent exprimer une «loyauté » en se conformant à une division sexuelle du travail prescrite par des rôles ou des normes de genre traditionnelles: Gershuny et al. (2005) proposent de nommer cette option "suffering » dans le cadre du travail domestique. Bien que ces modèles ne présentent pas d'hypothèses directement testables, ils introduisent l'idée que les ressources économiques importées dans la négociation interagissent avec des normes ou des identités de genre. De ce point de vue, Akerlof et Kranton (2000) ont énoncé l'idée qu'il y avait, pour des individus rationnels, un coût à s'éloigner de la norme, et que celui-ci pouvait être introduit à travers un paramètre d'« identité " au sein de la fonction d'utilité. II serait moins coûteux pour les conjoints de se comporter conformément à une division traditionnelle du travail domestique, que d'envisager une répartition plus égalitaire, car celle-ci entraine des coûts "psychologiques" importants. Akerlof et Kranton (2000) montrent que cette caractérisation introduit un effet non-linéaire et non-monotone dans la relation entre ressources économiques relatives et temps de travail domestique : lorsque la femme commence à gagner plus que son conjoint, elle aurait tendance à effectuer encore plus des tâches domestiques, menant à ce qu'Hochschild et Machung (1989) nomment le «supermoming ". Ces dernières ont en effet montré dans leur livre The Second Shift qu'au sein de multiples couples américains, l'amélioration de la situation économique relative des femmes ne se traduisait pas mécaniquement en une meilleure répartition des tâches domestiques au sein du couple. Selon elles, il faut élargir l'analyse pour prendre en compte ce qu'elles nomment les "économie maritales de gratitudes"; s'intéresser aux valeurs symboliques que les conjoints associent aux différentes tâches. Goffman (1979) fut le premier à proposer l'idée de " performance de genre " (Gender display dans le texte original ; le terme performance est souvent employé pour conserver l'aspect théâtral et cérémoniel du concept de Goffman), dans son livre Gender Advertisements : "[si] le genre est défini comme le corrélat culturellement établit du sexe (biologique ou acquis), alors la performance de genre renvoie aux représentations conventionalisées de ces corrélats " (op. cit., p 1, traduction de l'auteur de cet article). Si les époux français contribuent effectivement à « faire genre " (« doing gender », voir West et Zimmerman (1987)), ceci devrait avoir une conséquence sur la manière dont les ressources économiques interagissent avec les normes conventionnelles associées à la division sexuelle des tâches. Ceci peut s'exprimer en particulier dans les situations de déviance de genre où la femme contribue plus aux revenus du ménage que son mari (Bittman et al., 2003 ; England et Folbre, 2005). S'il est maintenant généralement accepté que les femmes travaillent et contribuent aux revenus du ménage, le modèle de l'homme pourvoyeur principal - le breadwinner -- reste la norme dominante, et le travail domestique peut devenir un mode de compensation symbolique dans ces situations déviantes, une forme de régulation de la vie conjugale. Si ces modèles introduisent une complexification intéressante liée à la prise en compte de processus sociaux ou normatifs plus fins, on peut dès à présent remarquer que le travail domestique n'est qu'une " scène " potentielle bien particulière de l'expression de la " performance de genre ". $\mathrm{Si}$ ce processus existe, on peut imaginer qu'il puisse s'exprimer par d'autres voies qu'une augmentation du temps consacré aux tâches domestiques (Schneider (2012), Hook (2015)) -- dans de multiples interactions quotidiennes, comme les pratiques verbales, sexuelles, dans la gestion des conflits, etc ${ }^{1}$. Ce n'est par ailleurs par la seule voie de compensation, Schneider (2012) a pu analyser ce type de phénomène dans le cas de situations d'emploi atypiques où les femmes ont un meilleur travail que leur mari et compenseraient par plus de travail domestique. L'article de Hook (2015) à paraître teste un modèle similaire au nôtre en comparant différentes situations déviantes qui pourraient mener les femmes à compenser en effectuant plus de tâches domestiques. Les effets sur la vie des couples du seuil au-delà duquel la femme gagne plus que son conjoint sont multiples, et s'étendent au-delà du travail domestique, comme l'a montré l'article récent de Bertrand et al. (2015). Ces auteurs mesurent l'impact du point de retournement sur des variables comme l'offre de travail des femmes, le nombre d'enfants dans le ménage, ou la probabilité qu'ont les couples mariés de divorcer. Et ceci bien que l'« aversion » des couples au fait que les femmes gagnent plus que leur mari (explication que les auteurs placent au cœur de leur travail) ne soit jamais prouvée ou analysée en tant que telle.

1. Ce n'est par ailleurs par la seule voie de compensation, Schneider (2012) a pu analyser ce type de phénomène dans le cas de situations d'emploi atypiques où les femmes ont un meilleur travail que leur mari et compenseraient par plus de travail domestique. L'article de Hook (2015) à paraître teste un modèle similaire au nôtre en comparant différentes situations déviantes qui pourraient mener les femmes à compenser en effectuant plus de tâches domestiques. 
relatives, qui devient, à partir d'un certain niveau de ressources, croissante dans le cas des femmes. Néanmoins, l'existence éventuelle de cet effet non linéaire et non monotone - cas (VI) - et son interprétation en termes de neutralisation d'une déviance de genre ${ }^{6}$ ont largement accaparé le débat empirique et théorique depuis l'article de Brines (1994). Cette manière extrême d'introduire une dimension symbolique dans l'analyse de l'influence des ressources économiques sur l'activité domestique a le défaut de vouloir mettre dos à dos une explication purement économique et une autre purement psychologique ou identitaire : d'un côté le salaire comme revenu, comme ressource matérielle, de l'autre le salaire comme ressource symbolique et cognitive permettant de situer les conjoints en négociation vis-à-vis des normes en vigueur. Le présent article a pour but d'ouvrir la possibilité d'une analyse moins tranchée. Pour ce faire, nous allons tester l'ensemble des " chemins » ci-dessous, en autorisant a priori les six possibilités identifiées.

La problématisation en terme de «NDG»a eu en effet tendance à présenter la forme non linéaire comme une caractéristique propre aux modèles « relatifs " - ce qui revient à réduire la déviance de genre à une anormalité dans les niveaux relatifs de salaire entre conjoints ${ }^{7}$. Or certains travaux récents (Gupta, 2006 et 2007 ; England, 2011; Sullivan, 2011; Killewald et Gough, 2010) ont cherché à montrer que l'effet de second ordre que l'on observe pour les ressources relatives était largement dû à une mauvaise prise en compte de la relation non linéaire entre niveau de salaire et temps de travail domestique, indépendamment du niveau relatif de salaire des conjoints.

\section{Les ressources économiques des femmes et le travail domestique}

\author{
Quelle caractérisation de la relation \\ entre salaire et temps de travail \\ domestique des femmes?
}

Les approches «absolue» et "relative» ont toutes deux été appuyées par des études empiriques dont les résultats sont loin de converger. Le premier article de Brines (1994) trouvait un effet linéaire des ressources relatives significatif pour les femmes et pas d'effet «NDG », et l'inverse apparaissait pour les hommes ${ }^{8}$. Greenstein (2000) a répliqué ces résultats en ayant recours à une mesure distributive du travail domestique - correspondant à la part du travail domestique total du ménage effectué par un individu : il montre l'existence d'un effet de second ordre, de type "NDG», significatif pour les deux conjoints. En travaillant sur des données australiennes, Bittmann et al. (2003) n'identifient aucun effet du salaire de la femme sur la quantité de travail domestique effectuée par l'homme, et trouvent à l'inverse un effet de premier et de second ordre du salaire de l'homme sur la quantité de travail domestique fournie par la femme?

\footnotetext{
6. Voir en particulier England (2011), Sullivan (2011) et Killewald et Gough (2010) pour une synthèse de ces débats.

7. De ce point de vue, l'article de Hook (2015) montre que la déviance de genre peut s'observer de différentes manières et n'opère pas uniquement par le biais du salaire des conjoints : le statut d'emploi (chômage, temps partiel ou temps plein) comme le type de métier exercé (Schneider, 2012) peuvent être d'autres manières d'observer l'influence de normes de genre issues de la sphère marchande, sur la sphère domestique.

8. Presser (1994) et Gupta (1999) reproduisent ces résultats pour les hommes, tout comme Ross (1987) qui met en évidence un profil inverse pour les femmes.

9. Il faut noter que l'effet NDG n'a jamais été observé simultanément pour les femmes et les hommes dans une même étude, voir Ponthieux et Meurs (2014).
}

\footnotetext{
Figure I

Ressources économiques et TD des femmes : approches et formes estimées

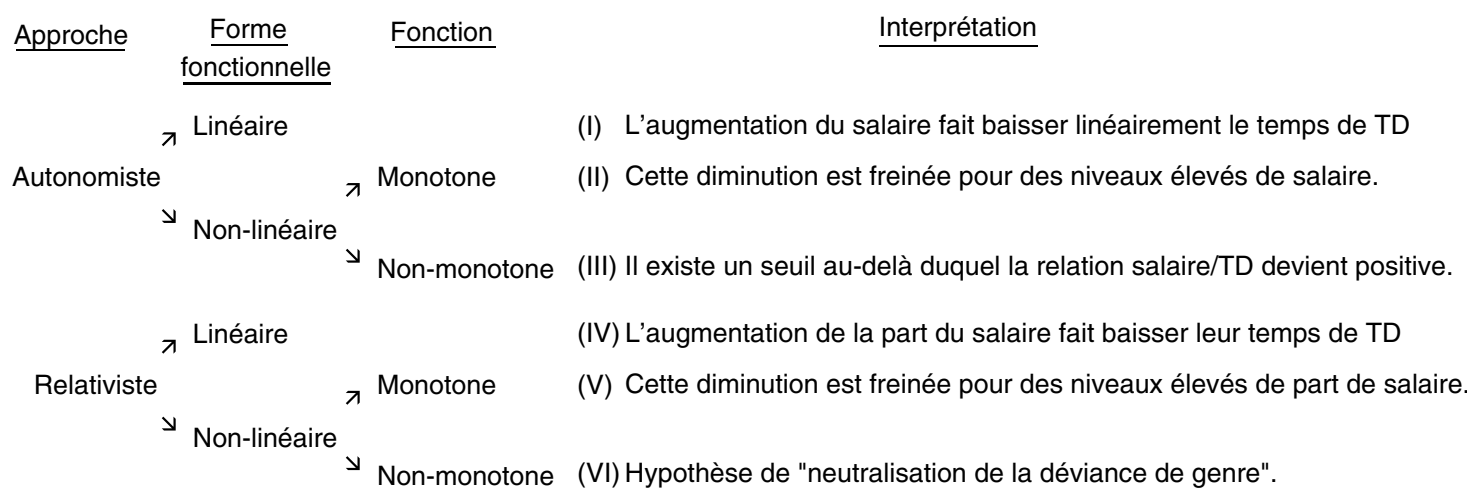


L'article récent de Bertrand et al. (2015) identifie, dans le cas des femmes américaines, un effet autonome pour les femmes lié au fait de gagner plus que leur mari, en contrôlant pour une mesure individuelle linéaire du salaire des conjoints.

Ces articles semblent donc à première vue valider empiriquement la thèse de la « NDG $»^{10}$, mais un ensemble d'autres travaux récents (Rizavi et Sofer, 2008 ; Gupta et Ash, 2008 ; Killewald et Gough, 2010; Sullivan, 2011) mettent en cause l'existence d'un tel effet. Gupta (2006 et 2007) a montré qu'en contrôlant correctement l'influence du salaire individuel dans le cas des femmes américaines, les effets (linéaire et non linéaire) des ressources économiques relatives sur les choix effectués par les femmes en matière de travail domestique disparaissaient. Ces articles, tout comme celui d'England (2011), affirment la validité de la thèse " absolue » et plaident pour une prise en compte des salaires autonomes des époux, plutôt qu'une mesure distributive de leurs ressources et du pouvoir de négociation qu'elle détermine. Dans la même perspective, Connelly et Kimmel (2009) n'ont trouvé qu'un effet très faible des salaires relatifs des conjoints sur la répartition des tâches au sein des ménages britanniques, et Rizavi et Sofer (2008) aboutissent au même résultat dans le cas de la France. Sullivan (2011), dans une synthèse de la littérature quantitative sur la question, affirme dans la même perspective que l'approche en termes de «NDG » est due à un surinterprétation statistique d'un phénomène qui, s'il existe, ne peut être prouvé que dans l'observation qualitative des parcours de vie. L'article de Lyonette et Crompton (2014) constitue l'un des premiers travaux qualitatifs développé dans la lignée de ces critiques, et ces deux auteures rejettent en effet très largement l'hypothèse de neutralisation de la déviance de genre. Ces auteures insistent beaucoup sur l'idée que ce phénomène de "NDG» ne concerne qu'un groupe très restreint de femmes, aux caractéristiques très spécifiques. Cependant, au sein des couples retenus dans notre étude, $36 \%$ des femmes ont un salaire mensuel supérieur ou égal à celui de leur mari : l'effet éventuel n'est donc pas a priori concentré sur une queue de distribution (cf. tableau 1) ${ }^{11}$.

Jusqu'à l'article de Killewald et Gough publié en 2010, la littérature a cherché à tester l'existence éventuelle d'un effet non linéaire des ressources relatives, mais en imposant systématiquement la linéarité dans le cas du salaire absolu. L'article de Bertrand et al. (2015) permet d'illustrer ce point dans un cas récent : ces auteurs autorisent des effets linéaires différents de part et d'autre du seuil fixé à $1 / 2$ pour la part du salaire total du ménage (le point au-delà duquel la femme gagne plus que son conjoint), mais cette non-linéarité n'est permise que pour les ressources relatives. Les auteurs n'incluent en particulier dans leur estimation qu'une mesure linéaire du salaire autonome ; comme si l'« identité de genre » ne pouvait opérer qu'à travers la question du positionnement économique relatif des conjoints. Sans remettre en cause l'intérêt et l'argument général de ce travail, il n'est pas sûr que l'indicatrice du seuil reste significative si l'on inclut dans l'estimation une mesure non linéaire du salaire des femmes.

Killewald et Gough (2010) ont en effet pu montrer que la quantité de travail domestique des femmes n'est pas également élastique au salaire à mesure que celui-ci augmente: lorsque les femmes gagnent plus sur le marché, elle vont certes avoir tendance à effectuer moins de travail domestique, mais cet effet de substitution va diminuer à mesure que leur salaire atteint des niveaux élevés. Indépendamment de la question du niveau de salaire différentiel entre conjoints, ou du pouvoir de négociation de chacun, pour des niveaux de salaire élevés, l'effet du salaire sur le temps consacré aux tâches domestiques par les femmes s'affaiblit, pour un ensemble de raisons économiques, sociales et psychologiques sur lesquelles nous reviendrons. Cela suggère que certaines tâches peuvent devenir incompressibles si les femmes souhaitent maintenir un degré d'implication dans le ménage, ou, de la même manière, que l'externalisation des tâches présente des coûts de transaction (recherche de services extérieurs, comparaisons entre services, contrôle et supervision, etc.) qui peuvent devenir trop importants à mesure que le recours aux services marchands s'accroît. Si tel est le cas, l'effet non linéaire observé pour la mesure des ressources économiques relatives ne serait qu'un artefact dû à une non-linéarité de l'effet du salaire seul.

La première partie de ce travail met en évidence des résultats similaires dans le cas de la France : il existe une relation non linéaire entre salaire et

10. L'article de Bertrand et al. (2015) ne se raccroche pas directement à cette littérature, mais le fait de choisir comme variable indépendante le fait de se situer à gauche ou à droite du seuil de $1 / 2$ de la part de salaire les en rapproche fortement; de même plus généralement que le statut central qu'ils accordent à ce seuil dans cet article.

11. L'article de Bertrand et al. (2015) montre qu'aux États-Unis il ne s'agit pas non plus d'un phénomène marginal. 
temps de travail domestique des femmes, mais la courbe estimée reste monotone, le retournement de celle-ci étant très largement extérieur au domaine d'estimation. Il semble exister une contrainte de positivité forte dans le cas du travail domestique des femmes qui expliquerait, au moins en partie, les difficultés qu'ont les femmes françaises à transformer plus de salaire en moins de travail domestique (Ponthieux et Schreiber, 2006). L'élasticité variable du travail domestique au salaire ne conduit pas à vérifier une hypothèse de type « NDG » dans le cas des ressources absolues (cas III), elle suggère plutôt l'existence de freins à la diminution du temps de travail domestique des femmes, qui peuvent être dus à des phénomènes de « compensation », mais également à un ensemble d'éléments sociaux, psychologiques, identitaires et économiques, dont l'identification causale dépasse le cadre de la présente étude. Ceci incite en première approche à différencier la question de la non-linéarité de celle de la monotonie de la relation identifiée, ce que ne fait pas le débat autour de la « NDG », qui s'intéresse à la non-linéarité uniquement à travers la question du retournement éventuel de la courbe.

\section{Substitution ou suppression de l'agenda des femmes?}

Nous ne chercherons pas ici à rendre compte des raisons précises, ou des processus de décision qui peuvent pousser les femmes à déléguer, externaliser ou supprimer certaines tâches de leur emploi du temps; notre objectif sera avant tout descriptif, et cherchera à caractériser empiriquement la forme fonctionnelle de la relation entre le salaire des femmes françaises et le temps de travail domestique de celles-ci, en insistant sur des effets différenciés par types de tâches. Il s'agira donc, dans la seconde partie de l'article, de décrire plus en détail cette nonlinéarité, en tentant de répondre à deux questions qui n'ont pas été posées par les études récentes. Sur quelle tâche ou ensemble de tâches ces effets portent-ils et que devient ce temps non-effectué par les femmes ? Nous montrerons que les tâches par lesquelles s'effectuent cette diminution sont avant tout la cuisine et le ménage ${ }^{12}:$ les femmes choisissent de consacrer moins de temps à ces activités à mesure que leur revenu augmente. Le temps consacré aux enfants par les femmes est à l'inverse insensible aux modifications des revenus, un résultat déjà mis en évidence par Bloemen et Stancanelli (2008), Guryan et al. (2008). Trois possibilités s'offrent alors aux couples: premièrement, les hommes peuvent prendre à leur charge ces tâches " déléguées 》 par les femmes, ceci s'observe avant tout dans le cas du ménage, et dans une moindre mesure de la cuisine. Une seconde alternative peut être d'externaliser ces tâches hors du ménage par un recours à des services marchands équivalents, solution que la littérature anglo-saxone qualifie de «buy out » (Killewald, 2011). Néanmoins, nos données montrent dans l'ensemble que l'effet d'un recours à des services extérieurs sur le temps consacrés aux mêmes tâches en interne est faible ${ }^{13}$, à l'exception du ménage et du recours aux aides ménagères.

Nous montrerons ainsi que ces deux mécanismes de substitution ne compensent pas entièrement le temps mis de côté par les femmes à mesure que le salaire augmentent, un résultat suggéré par l'étude de Ponthieux et Schreiber de 2006. Ces constats montrent en effet que ce qu'on modélise n'est pas directement un volume fixe de tâches à se répartir, et ceci plaide dans l'ensemble pour une meilleure prise en compte des mécanismes spécifiques à chaque tâche et d'avantage d'études qualitatives sur le sujet. Nous suggérerons une explication possible, face à des salaires plus élevés, les ménages peuvent choisir de modifier la production domestique par sa « marge extensive » : le volume de tâches global diminuerait, menant, selon Killewald (2011), à une diminution de la «qualité » de la vie domestique ${ }^{14}$. À mesure que les femmes consacrent moins de temps au travail domestique, certaines tâches ne seraient simplement plus effectuées ou elles seraient réalisées avec un investissement en temps réduit : le ménage et la cuisine seraient en particulier faits plus rapidement, avec moins d'attention ou de soin. Cette hypothèse est a pu être mise en avant dans la littérature quantitative (Killewald, 2011 ; Craig et Baxter, 2014), mais les appuis empiriques qualitatifs, qui la soutiennent sont souvent faibles ${ }^{15}$. Il n'existe pas à notre connaissance à ce jour d'étude portant précisément sur les implications de ces modifications par la « marge extensive » de la production domestique, et il convient de rester prudent quant à l'interprétation que nos données permettent de formuler. Les travaux portant sur des tâches spécifiques sont en effet

12. Ceci corrobore les résultats de Hook (2010).

13. Ceci est appuyé par de nombreux travaux récents, voir en particulier Killewald (2011), Sullivan et Gershuny (2013) et Craig et Baxter (2014).

14. Cette solution est qualifiée de "opt out » dans la littérature, voir Killewald (2011).

15. On trouve des éléments à l'appui de cette hypothèse, en particulier dans l'étude sur le couple Tanagawa (Hochschild et Machung, 1989, chapitre 6), ou dans Bereni et al. (2011), mais ces éléments sont souvent à la marge des études conduites. 
moins courants dans la littérature, ceci étant avant tout dû au fait que les données récoltées restent souvent agrégées et récoltées a posteriori. Néanmoins, l'article de Hook (2008) portant sur une comparaison internationale a pu montrer que les femmes françaises modifient le temps passé à préparer la cuisine et à s'occuper des repas, ainsi que celui passé à faire le ménage. L'auteure trouve que les hommes français réagissent à cette baisse en prenant à leur charge une partie des tâches liées au ménage, mais très peu celles liées à la préparation des repas.

Nous suivons ainsi la ligne défendue par Sullivan (2011) et England (2011), en tentant de recentrer l'analyse quantitative sur ce qu'elle permet de saisir, à savoir la description macrosociale d'effets nécessairement inspirés de travaux qualitatifs, mais qui ne cherche pas à se substituer à ces travaux dans la compréhension des raisons profondes de ces effets ${ }^{16}$.

\section{Données et mesure}

\section{L'enquête Emploi du temps et l'échantillon retenu}

Nous nous appuyons sur les données issues de l'enquête Emploi du temps 2009-2010 afin de tester la validité de ce modèle dans le cas de la France actuelle. La structure de l'enquête permet de limiter les biais liés à l'évaluation ex post par les individus de la quantité de travail domestique qu'ils fournissent ${ }^{17}$. De nombreuses enquêtes s'appuient en effet sur une question agrégée quant à la quantité de travail domestique effectuée la semaine précédant l'enquête, ce qui conduit à une mesure extrêmement imprécise des tâches effectuées (parmi ces enquêtes on peut mentionner la Panel Study of Income Dynamics ou le National Survey of Family and Households). Le biais principal est dû au fait que les conjoints ont tendance a posteriori à surrévaluer la quantité de travail domestique qu'ils effectuent (Coltrane, 2000 ; Marini et Shelton, 1993), et ce phénomène est proportionnellement plus prononcé pour les hommes (Townlsey et al., 1998), ceci réduisant artificiellement les inégalités mesurées dans la répartition des tâches. L'article de Killewald et Gough (2010), s'appuie sur une mesure renseignée ex post par les individus, et un apport de notre travail consiste à tester la validité de leurs résultat sur des données plus précises ${ }^{18}$. L'apport du présent article vis-à-vis de cette étude consiste à évaluer si le résultat original mis en avant peut être dû à un biais lié à une mauvaise mesure du travail domestique. En effet, si les individus évaluent subjectivement la quantité de temps qu'ils ont allouée au travail domestique la semaine de l'enquête, il n'est pas impossible qu'on observe un effet similaire de « performance » au niveau de la réponse donnée. Ainsi, les femmes, par rapport aux hommes, sous-évaluent en général la quantité de travail domestique qu'elles effectuent, et il paraît délicat de contrôler correctement l'effet d'une éventuelle « performance de genre » qui peut se jouer au moment du report (Sullivan, 2011, avait déjà souligné ce point). L'effet de compensation mis en évidence par Brines (1994) et Bittman et al. (2003) peut être estompé, ou au contraire accentué par les femmes au moment de l'évaluation de leurs temps de travail domestique; il existe à notre connaissance peu d'éléments qui permettent d'anticiper le signe de ce biais. Il est tout à fait possible que l'effet de « NDG » soit un pur produit du moment de réponse à l'enquête, sans que les femmes ne fassent en réalité plus de tâches domestiques. À l'inverse, il est envisageable, si ce processus de performance n'est pas tout à fait conscient, que l'écart d'évaluation entre les hommes et les femmes quant à la quantité de travail qu'ils effectuent augmente, pour des niveaux élevés de revenus relatifs. En résumé, il pourrait y avoir une non-linéarité dans le biais lié à une mesure a posteriori du travail domestique. Cela expliquerait que l'effet dû à une " performance de genre » disparaisse dans l'estimation de Killewald et Gough (2010), et le recours aux données de l'enquête Emploi $d u$ temps nous permet d'examiner cette éventualité.

Dans l'enquête Emploi du temps, chaque individu enquêté doit en effet indiquer une activité

16. Les travaux visés par ces critiques sont avant tout ceux qui infèrent de résultats ou de paramètres quantitatifs des conclusions générales sur la persistance ou l'importance des " normes » ou " identités » de genre, sans que ces notions générales ne soient précisément interrogées, et sans que la littérature qualitative sur le sujet ne soit discutée, au-delà de la référence au livre d'Hochschild et Machung (1989). Voir en particulier Akerlof et Kranton (2000) et Bertrand et al. (2015).

17. Sur ce problème, voir en particulier Hill et Stafford (1980), Bianchi et al. (2000) ou encore Pallié (1993) et Niemi (1993).

18. Un doute lié aux carnets journaliers concerne la représentativité du jour de l'enquête. En dehors de la question de la spécificité d'un jour de semaine, il se peut qu'il y ait du bruit lié à la particularité du jour de l'enquête. La base Emploi du temps contient une variable qui indique si le jour d'enquête est représentatif d'une journée typique de l'individu: dans notre échantillon, $89 \%$ des femmes interrogées indiquent que les activités essentielles de la journées ont été correctement renseignées, et ce pourcentage est le même pour les hommes. Nous avons fait le choix de ne pas restreindre l'échantillon à ces femmes, et ceci ne supprime pas complètement le problème de représentativité. Cependant, cette variable subjective indique qu'une grande majorité des enquêtées est satisfaite de la manière dont les activités ont été reportées, et qu'il n'y a pas de différences majeures entre hommes et femmes dans la manière dont ils évaluent la qualité du carnet. 
principale et une activité secondaire pour chaque tranche de 10 minutes durant la journée d'enquête, il y a donc 288 entrées par individu. Tous les individus enquêtés ont rempli un carnet pour un jour de semaine, et $42 \%$ des individus ont rempli un second carnet pour un jour de week-end. Des informations complémentaires portant sur les caractéristiques socio-économiques (salaire, âge, situation conjugale, niveau d'éducation, etc.) des individus sont également disponibles, et la combinaison des deux types de variables rend cette base particulièrement adaptée à notre questionnement. La plupart des enquêtes statistiques fondées sur les emplois du temps ne comportent pas, en effet, de variables socio-économiques complémentaires et obligent les chercheurs à construire des proxies, en particulier pour estimer les niveaux de salaire ${ }^{19}$.

Nous avons restreint notre analyse aux couples hétérosexuels bi-actifs occupés, exerçant une activité salariée à temps complet, en situation de cohabitation $^{20}$. Nous avons éliminé les couples où l'un des invididus est retraité ou n'exerce pas d'emploi ${ }^{21}$, où l'un des conjoints est en institution ou en situation de handicap. Une autre manière de procéder, suivie par Solaz (2005), aurait été de considérer l'influence des période de chômage sur la répartition du travail domestique. Solaz montre en effet que les maris subissant une période de chômage ne prennent pas à leur charge autant de travail domestique que ce que prédisent les modèles microéconomiques ; ce sont avant tout les tâches typiquement féminines qui sont les plus aisément transférables. Le statut matrimonial influence fortement la manière dont les couples vivent et conçoivent la répartition des tâches domestiques, que les conjoints soient mariés (De Singly, 1987) ou en cohabitation (Domingues-Folgueras, 2013). Restreindre notre échantillon aux couples en cohabitation permet d'une part de simplifier l'interprétation des résultats en termes de logique interne au ménage, et d'autre part de pouvoir comparer nos estimations à celles de la littérature sur le sujet, qui portent presque exclusivement sur ce type de couple.

Le choix de ne s'intéresser qu'à ces couples occupés, salariés à temps plein permet de restreindre notre analyse à des individus ayant le même statut d'emploi, et ainsi de comprendre précisément l'impact des ressources économiques sur la répartition des tâches domestiques. Les études portant sur des couples à statut d'emploi différent contrôlent l'effet de ce statut par une mesure du temps consacré au travail marchand, afin de raisonner à temps de travail égal (Brines,
1994). Mais comme l'ont indiqué Killewald et Gough (2010), cette méthode ne fonctionne que si l'on modélise correctement la relation entre salaire et temps de travail marchand. En particulier, les revenus mensuels des conjoints sont liés au nombre d'heures travaillées, et le niveau de salaire lui-même dépend des investissements en capital humain(Becker, 1985), ce quirendl'interaction entre les deux difficile à saisir. Cette endogénéité dans les choix de statuts d'emploi ${ }^{22}$, en particulier des femmes, introduit une hétérogénéité dans l'échantillon qui rend complexe la prise en compte des biais existant entre ressources économiques et statut d'emploi ${ }^{23}$. Nos estimations principales portent sur les carnets remplis un jour de semaine. Nous indiquons néanmoins en annexe 1 des statistiques descriptives portant sur les jours de week-end : le temps consacré aux tâches domestiques, globalement et par tâche, est très proche des résultats portant sur les jours de semaine. Il est légèrement supérieur pour le volume global mais les écarts entre hommes et femmes sont maintenus : au regard de nos données, il n'y a pas de spécificité des jours de week-end (voir annexe 1, tableau 6), les tâches spécifiques comme le ménage ou le linge n'y sont pas effectuées en priorité ${ }^{24}$.

Notre base ainsi constituée comprend 3348 observations, 1674 femmes et 1674 hommes, et le tableau 1 fournit des statistiques descriptives quant à l'échantillon considéré.

19. La grande enquête par emplois du temps aux États-Unis, la Panel Studies of Income Dynamics présente cet écueil majeur, et conduit certains auteurs comme Bittman et al. (2003) à décider de ne pas y avoir recours.

20. Voir Courduriès (2008) pour une analyse portant sur les couples homosexuels.

21. L'effet du passage à la retraite entraine des modifications de la répartition des tâches domestiques au sein des couples francais (Solaz, 2005), mais les logiques qui y président sont a priori différentes de celles liées à l'exercice d'une activité salariée qui nous intéressent ici.

22. Ici entre temps complet et temps partiel.

23. Notre estimation principale concernant la déviance de genre compensatoire est néanmoins robuste à un choix d'échantillon différent : nous avons testé nos modèles en incluant les femmes à temps partiel, les hommes à temps partiel, et l'ensemble des conjoints à temps partiel. L'effet non-linéaire du salaire absorbe l'effet non-linéaire de la part de salaire quel que ce soit l'échantillon retenu. De surcroît, la valeur des coefficients principaux portant sur les variables indépendantes-salaire de la femme et salaire quadratique de la femme - n'est que très faiblement modifiée (entre $4 \%$ et $7 \%$ ), et le degré de significativité est le même pour des échantillons de population plus large, incluant les individus à temps partiel. Notre base de données montre un écart de 15 points de pourcentage entre le taux de salariés en couple bi actif occupés à temps partiel des hommes (6,7\%) et celui des femmes $(21,7 \%)$.

24. Nous avons également testé l'ensemble de nos modèles pour l'échantillon des carnets remplis un jour de week-end, et les résultats restent proches pour ce qui est des coefficients de régression. Il y a deux fois moins d'individus dans cet échantillon, les écarts-types varient en conséquence davantage, mais les principaux résultats en termes de significativité sont vérifiés pour les jours de week-end. 
Au sein des couples étudiés, les hommes effectuent moins de travail domestique que les femmes ${ }^{25}$, et cet écart diffère selon la tâche observée. La répartition de la cuisine et des tâches ménagères est plus inégalitaire que le volume global, ceci s'expliquant en partie par une allocation plus égalitaire du temps passé à s'occuper des enfants. Laver et ranger le linge n'a occupé qu'une minute et 20 secondes de la journée des hommes enquêtés, ce qui explique les différences massives avec le temps qu'y consacrent les femmes. On constate enfin que les femmes salariées à temps plein gagnent $28 \%$ de moins que leur conjoint et qu'elles travaillent en moyenne une heure de moins le jour de l'enquête.

\section{Le temps de travail domestique}

La mesure du travail domestique est un élément clé du travail de recherche sur des données de type emploi du temps ${ }^{26}$ : plusieurs agrégats ont été proposés par Brousse (1999) et par Roy (2011), qui renvoient à des acceptions plus ou moins larges de ce que recouvre le « travail domestique ». Plus on étend le cercle des tâches incluses dans l'ensemble des tâches domestiques, plus l'écart entre les hommes et les femmes se réduit. Le travail domestique est plus difficile à cerner que le travail marchand : certaines tâches (faire le ménage, la cuisine, s'occuper de l'hygiène des enfants, etc.) sont davantage marquées socialement comme étant du «travail» au sens de « peine » et sont avant tout effectuées par les femmes, alors que d'autres, plus proches du loisir ou du semi-loisir (faire du bricolage, promener le chien, jouer avec les enfants, etc.), sont mieux équitablement réparties entre les époux. Nous avons décidé dans cet article de garder une définition restreinte des tâches domestiques, l'ensemble des tâches retenues est résumée dans le tableau 2 . Nous y incluons également la définition des quatre postes spécifiques que sont la cuisine, le ménage, le linge et le temps passé à s'occuper des enfants. Le poste «faire la cuisine » exclut en particulier la préparation exceptionnelle de la cuisine, ou la cuisine de réception, plus souvent prises en charge par les hommes que la cuisine de tous les jours. La définition du « childcare», ou temps consacré à s'occuper des enfants, est plus complexe car elle renvoie à un ensemble d'activités très diverses qui incluent l'hygiène ou les soins apportés, la supervision et les tâches éducatives, le jeu ou encore le transport; nous avons fait le choix de restreindre notre analyse aux tâches les plus facilement identifiables à une charge domestique, à savoir l'hygiène, les soins et les tâches de supervision. Il existe un débat important dans la littérature quant à la question d'inclure ou non le temps consacré aux enfants dans la mesure du travail domestique (Sullivan, 2013). Un problème émerge en particulier lorsqu'on fait face à une mesure agrégée ou indiquée ex post, car il est alors impossible de distinguer précisément l'ensemble de tâches réalisé par les conjoints, ce qui pousse de nombreux travaux américaines à exclure l'ensemble du «childcare ». L'enquête Emploi du temps nous permet de sélectionner des sous ensembles de tâches au sein de chaque poste et nous avons fait le choix de retenir certains tâches liées aux enfants, plus aisément identifiables à des corvées domestiques (cf. tableau 2$)^{27}$.

\footnotetext{
25. Le détail de la mesure agrégée du travail domestique est expliqué ci-après.

26. Bien entendu, les travaux reportant une mesure agrégée ne permettent pas de travailler directement sur ce problème.

27. La base Emploi du temps ne permet cependant pas de sélectionner des tâches spécifiques. II est impossible de savoir, par exemple, ce que recouvre précisément le sous poste "s'occuper des enfants à domicile", alors que des logiques genrées peuvent apparaitre au sein de tâches regroupées dans cet ensemble agrégé : entre "donner le bain » et "changer les couches " par exemple. Avoir plus de détails pour ce poste précis permettrait d'affiner l'analyse, mais nous sommes déjà en mesure d'exclure les tâches plus proches du semi-loisir comme "faire la conversation ", " jouer, câliner », etc.
}

Tableau 2

La mesure du temps de travail domestique dans l'enquête EDT

\begin{tabular}{|l|l|}
\hline Niveau d'agrégation & \multicolumn{1}{c|}{ Activités } \\
\hline Volume global & $\begin{array}{l}\text { Inclut les postes Ménage, Cuisine, Linge, S'occuper des enfants, transport non professionnel } \\
\text { pour le ménage, les tâches administratives, comme s'occuper des papiers, et les soins à l'adulte. } \\
\text { Préparation et cuisson des aliments, épluchage, mettre la table, servir le repas. } \\
\text { Lavage de la vaisselle, rangement de la vaisselle, débarrasser la table, rangement des courses, chargement } \\
\text { et déchargement de la voiture, rangement et nettoyage extérieur, rangement et nettoyage intérieur. } \\
\text { Lavage du linge (le trier, le mettre dans / sortir de la machine à laver, l'étendre), repassage, couture, tricot, } \\
\text { crochet, cirage et lavage des chaussures. } \\
\text { S'occuper d'enfants de son ménage, accompagner un enfant de son ménage, l'attendre (hors trajets), } \\
\text { procurer des soins médicaux à domicile, surveillance des devoirs et leçons. }\end{array}$ \\
\hline
\end{tabular}


D'autre part, l'idée sous-jacente aux modèles « relatifs » est que le ménage doit se répartir un volume de tâches fixes à la suite d'un processus de négociation entre conjoints. Nous avons choisi ici d'adopter une position plus neutre et de fixer comme variable dépendante le volume absolu de tâches effectué par la femme, et non la part du volume total prise en charge, laissant la possibilité d'avoir un temps domestique autonome par conjoint.

\section{Variables de contrôle}

Nous incluons dans notre modèle différentes variables de contrôle sans lesquelles l'analyse qui suit pourrait être faussée ${ }^{28}$. L'âge et l'âge au carré sont introduits pour neutraliser la présence d'attitudes différentes liées au positionnement dans le cycle de vie (Brines, 1994). Nous introduisons 4 variables binaires liées au niveau de diplôme le plus élevé, $\mathrm{Bac}+5$ ou plus, $\mathrm{Bac}+3$, Baccalauréat et brevet des collèges; le groupe de référence constitue l'ensemble des non-diplômés de l'échantillon. Le niveau de revenu est fortement corrélé au niveau d'éducation, mais ce dernier peut également influencer la quantité de travail domestique faite par les individus, introduisant ainsi un biais potentiel. Nous introduisons le nombre d'enfants présents dans le ménage, de la naissance à la majorité, car ceux-ci augmentent mécaniquement la quantité de travail domestique faite par les époux, ainsi que les inégalités entre conjoints: l'effet d'un enfant supplémentaire sur le temps de travail domestique des femmes est plus important que sur celui des hommes (Pailhé et Solaz, 2008 ; Brines, 1994 ; Baizàn et al., 2014). Une dernière variable liée au lieu de résidence est introduite : les individus sont regroupés entre trois catégories, ceux qui résident au sein ou en périphérie de grand pôles urbains, au centre au en périphérie de pôles urbains de taille moyenne, et le groupe de référence qui réside en zone rurale ou autour de petits pôles urbains. La situation du bassin d'emploi de résidence peut influencer le niveau de revenu des individus-les salaires étant en moyenne plus élevés autour des grands pôles urbains - mais également les attitudes des ménages envers la répartition du travail domestique entre conjoints. On peut se référer aux travaux de Bessière (2004 et 2008) pour des éléments spécifiques à la gestion des tâches domestiques dans les familles d'agriculteurs ou à l'inverse, à Bereni et al. (2011) pour une étude portant sur les femmes de la haute fonction publique parisienne.

\section{L'effet du salaire des femmes sur leur temps de travail domestique}

\section{Un effet négatif, non-linéaire et monotone du salaire des femmes}

Le grand nombre d'observations présentant une quantité nulle de travail domestique suggérerait l'utilisation de modèles adaptés à des données tronquées. Néanmoins, les techniques habituelles pour gérer des données censurées (modèles tobit ou heckit) sont aujourd'hui considérées comme moins précises que des régressions linéaires pour ce qui est des données de type emploi du temps (Stewart, 2013) ${ }^{29}$. Nous avons recours à un modèle linéaire estimé pour les femmes occupées à plein temps sur un agrégat restreint de tâches défini précédemment et corrigeons l'hétéroscédasticité du modèle en estimant les écart-types par cluster au niveau du ménage. Le premier modèle (1) se situe dans une perspective "relative", et utilise comme variables indépendantes la part de salaire, ainsi que la part de salaire quadratique, le salaire de la femme et le salaire du conjoint, et en contrôles les variables définies ci-dessus, il teste donc les cas (I), (IV), (V) et (VI) de la figure I. La part de salaire est mesurée par le niveau de salaire d'un individu divisé par la somme des salaires des deux conjoints ${ }^{30}$. Nous n'incluons pas le revenu total du couple pour des raisons évidentes de colinéarité.

On observe dans ce cas (modèle (1)) un effet, de premier et de second ordre, de la part de salaire, et un effet linéaire du salaire de la femme. Il y a donc bien un effet négatif autonome du salaire des femmes sur leur temps de travail domestique, et l'effet de la part de salaire change de signe suivant le niveau de salaire auquel on se situe. La présence du terme quadratique fait

\footnotetext{
28. La question des variables de contrôle a également fait l'objet de nombreux débats dans le domaine, et nous avons recours à la spécification la plus généralement acceptée aujourd'hui, celle de Gupta (2007).

29. La contrainte de positivité du travail domestique n'en reste pas moins un élément important à garder l'esprit: le rapport entre le travail domestique et ses variables déterminantes risque d'adopter une forme non linéaire de par cette contrainte, et le type de non-linéarité (quadratique ou continue par morceaux) qu'on cherche à capturer s'en retrouvera influencée. 30. Le nombre de valeurs manquantes que cela crée est nul pour notre échantillon. Prendre comme mesure distributive le rapport de salaire (et non pas la différence), est cohérent avec la littérature sur le sujet (inspirée des conditions de premier ordre mentionnées ci-dessus), et permet de conserver à la fois le salaire de l'individu et celui de son conjoint, sans soucis de colinéarité. Voir en particulier Bertrand et al. (2015) pour une utilisation récente de cette mesure.
} 
de l'effet de la part de salaire des femmes une fonction non linéaire de cette part de salaire et le signe de cet effet dépend de la position par rapport au point de retournement. L'inversion se produit pour un niveau de part de salaire de $235,47 /(2 \times 172,87)=0,68$, en d'autres termes l'effet non linéaire n'est positif que pour les femmes qui gagnent plus de deux-tiers des revenus du travail du ménage. Le premier graphique de la figure II représente la relation entre part de salaire et temps de travail domestique telle que prédite par l'estimation de notre premier modèle. Seules 48 femmes de notre échantillon se situent au-delà du seuil de retournement, soit 2,8\% de l'ensemble des femmes: sans être extérieure au domaine d'estimation, l'inversion est cependant fondée sur un très petit nombre d'observations. Il s'agit d'un élément rarement mis en avant par les travaux s'intéressant à la thèse «NDG »; or il remet directement en cause ses conclusions. D'une part le point de retournement ne se situe pas au seuil symbolique de 0,5 (Akerlof et Kranton, 2000 ; Bertrand et al., 2015), et d'autre part l'effet positif identifié est quasi inexistant lorsqu'on le rapporte aux données de l'échantillon. À titre de comparaison, le seuil calculé à l'aide des estimations de Killewald et Gough (2010) se situe à un niveau de de $84 \%$ des revenus du ménage, ce qui incite à croire que le retournement a également de grandes chances d'être extérieur au domaine d'estimation dans leur cas. À mesure que le niveau de salaire augmente, la quantité de travail domestique effectuée par les femmes diminue, et à niveau de salaire équivalent, les femmes qui gagnent relativement plus que leur maris parviennent à faire peser le processus de négociation en leur faveur, à l'exception de celles qui gagnent plus de 2,13 fois le salaire de leur mari. On observe donc bien un effet non linéaire positif des ressources relatives, ce qui semble aller dans le sens de Greenstein (2000), Bittman et al. (2003) et Bertrand et al. (2015), mais cet effet concerne un nombre très limité de femmes.

Il faut également noter que l'effet du salaire sur la diminution du temps de travail domestique est faible, puisque, tel que mesuré par notre premier modèle, 100 euros de plus équivalent à 1 minute et 24 secondes de moins de travail domestique. La part de la variance du travail domestique des femmes expliquée par notre modèle est d'environ $19 \%$, en comparaison elle se situe autour de $11 \%$ pour les hommes (modèle (4)).

Le second modèle s'inspire des travaux de Killewald et Gough (2010) et introduit une mesure non linéaire du salaire des femmes, carrée dans le modèle (2) et linéaire par morceaux dans le modèle (3); il teste les six cas mentionnés par la figure 1. La caractérisation linéaire par morceaux est habituellement retenue dans la littérature, mais le nombre relativement faible d'observations dans notre cas fait que nous avons préféré insister sur une mesure quadratique du salaire (2), moins précise dans l'estimation de la forme fonctionnelle mais qui évite de diviser l'échantillon dans l'estimation. L'estimation à l'aide d'une fonction linéaire par morceaux (spline linéaire) pose également la question du choix des nœuds, qui reste encore arbitraire dans la littérature: Killewald et Gough (2010) utilisent une estimation par quartiles, alors que Hook (2015) s'appuie sur des ruptures aux cinquième et neuvième déciles. Dans notre cas, les résultats des estimations linéaires par morceaux ne sont pas robustes au choix des points, bien que dans l'ensemble des spécifications l'effet de second ordre des ressources relatives disparaisse.

Nous avons choisi de présenter les résultats par quartiles $^{31}$, dans un esprit de comparaison avec les travaux de Killewald et Gough (2010).

Dans ces deux modèles, l'effet de premier et de second ordre de la part de salaire des femmes disparaît, ce qui montre bien les limites de la caractérisation utilisée dans le premier modèle. Les deux modèles (2) et (3) montrent que pour des salaires plus élevés, et à pouvoir de négociation équivalent, le temps que les femmes consacrent au travail domestique diminue. Mais cette substitution est freinée par un effet non linéaire, la quantité de travail domestique effectuée par les femmes ne diminuant pas uniformément à mesure que le salaire augmente. Dans le modèle (2), à part de salaire égale, 100 euros de revenus supplémentaires ont pour résultat une baisse de 4 minutes et 36 secondes du temps de travail domestique pour les femmes ${ }^{32}$. Nous reproduisons ainsi les résultats de Killewald et Gough (2010) dans le cas des ménages français, à la différence que nos données sont issues de carnets précis et non de mesures indiquées

31. Nous avons également testé ce modèle selon l'approche de Hook (2015) avec deux points aux 5ème et 9ème déciles, puis en divisant l'échantillon en déciles. Enfin, nous avons testé une approche avec un point de rupture au SMIC net d'une valeur de 1050,63 euros mensuels à la fin 2009, et un second à deux fois le SMIC net. Ces estimations font toutes disparaître l'effet non-linéaire de la part de salaire, mais ne donnent pas de résultats robustes.

32. Ces résultats sont difficilement comparables numériquement avec ceux de Killewald et Gough (2010), car ces auteures raisonnent sur des revenus annuels et un temps de travail domestique hebdomadaire. 
a posteriori : leur résultat original sur la disparition de l'effet quadratique n'est semble-t-il pas dû à une mauvaise mesure du travail domestique, ce que nous cherchions à vérifier. La représentation graphique des prédictions des modèles (2) et (3) (cf. figure II) montre que cette augmentation du salaire des femmes ne les conduit pour autant pas à « compenser » en effectuant plus de tâches domestiques, la courbe estimée est monotone : c'est bien le cas (II) qui est validé par ces modèles. Le temps de travail domestique diminue effectivement à mesure que leur salaire s'accroît; simplement, cette augmentation est freinée pour des niveaux de salaire élevés. Nous avons testé les modèles (1), (2) et (3) en remplaçant la mesure quadratique de la part de salaire par une autre mesure non linéaire des ressources relatives, dichotomique prenant la valeur 1 si l'individu gagne plus que son conjoint. Il s'agit de la non-linéarité retenue par Bertrand et al. (2015) et cette estimation donne des résultats très proches de ceux du tableau 3 portant sur la part de salaire : l'effet du seuil disparaît lorsqu'on introduit une mesure non linéaire du salaire des femmes.

Dans le modèle (2), le retournement de l'effet du salaire des femmes se produit pour un niveau de salaire de 4406 euros mensuels (cf. figure II), ce qui concerne 21 femmes de notre échantillon. Ce modèle (2), qui s'appuie sur une spécification quadratique du salaire, ne met pas donc plus en évidence de mécanisme de compensation pour des niveaux élevés de salaire. Le point de retournement étant à la limite du domaine d'estimation, il semble que la spécification linéaire par quartiles utilisée dans le modèle (3) caractérise mieux la non-linéarité à laquelle nous faisons face. Dans le modèle (3), on voit que la valeur absolue du coefficient diminue entre le premier et le quatrième tronçon de la courbe ${ }^{33}$, ce qui montre bien - malgré la non-significativité pour les deuxième et troisième quartiles - que l'élasticité entre travail domestique et salaire diminue à mesure que celui-ci augmente. L'effet d'une augmentation de 100 euros sur le temps de travail domestique des femmes est de 8 minutes 48 secondes pour le $1^{\text {er }}$ quartile de revenu, alors qu'il n'est que de 1 minute 30 secondes pour le $4^{\mathrm{e}}$ quartile.

33. Nous n'avons représenté les prédictions des effets marginaux uniquement pour le premier et le quatrième quartiles de salaire (partie droite de la figure 2), pour lesquels l'effet mesuré est significatif.

Figure II

Prédictions du temps de travail domestique des femmes

A - En fonction de la part de salaire

$B$ - En fonction du salaire
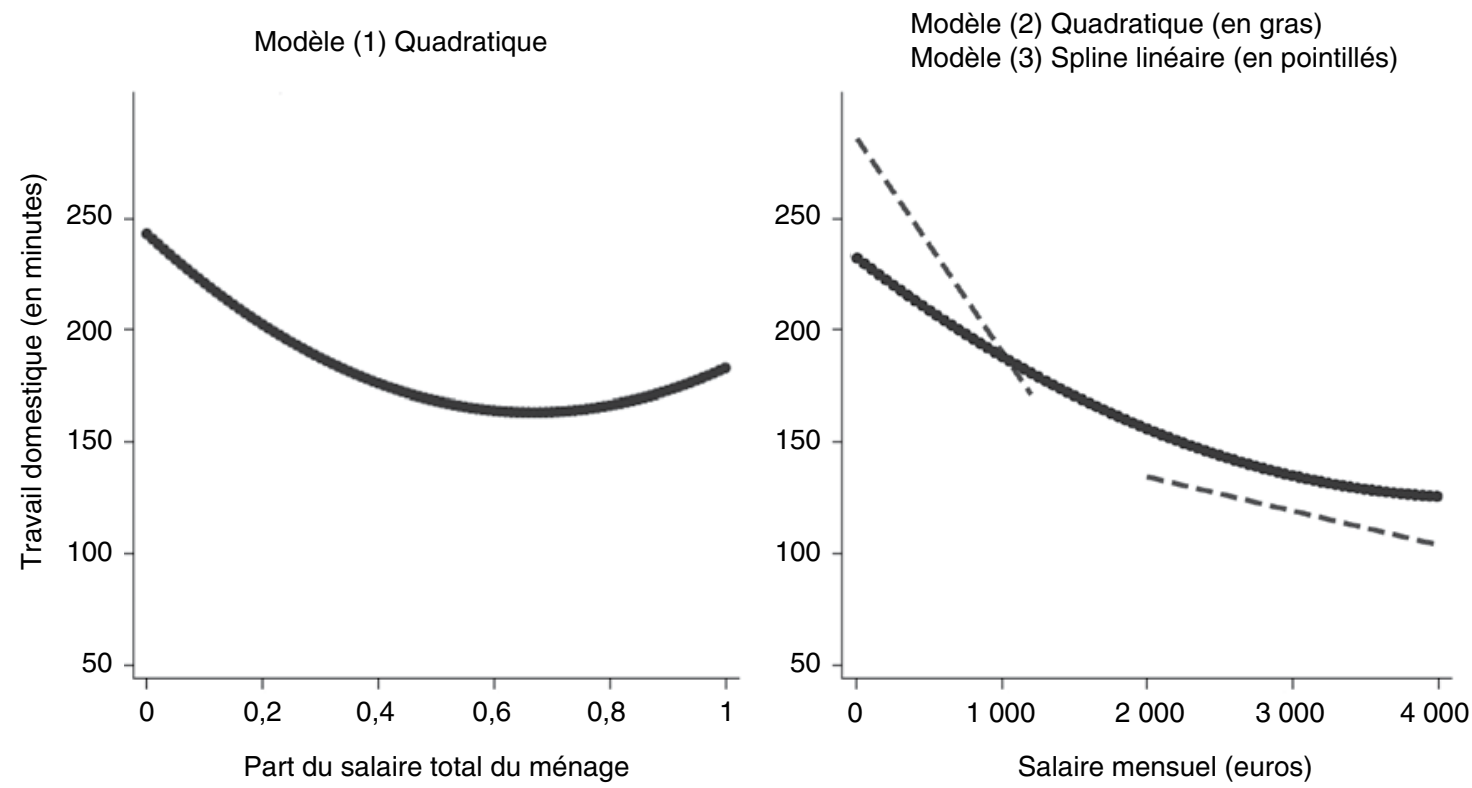

Lecture : ces courbes représentent les effets marginaux conditionnels prédits par les modèles (1), (2) et (3). Les paramètres estimés sont la constante mesurée dans le tableau 3, corrigée de la moyenne conditionnelle des variables du modèle. Pour les modèles (1) et (2), les effets marginaux sont obtenus en combinant les coefficients de regression linéaires et quadratiques de la part de salaire et du salaire respectivement. Nous ne représentons que les quartiles pour lesquels l'effet mesuré est significatif dans le cas du modèle (3). Champ : couples hétérosexuels bi-actifs occupés, exerçant une activité salariée à temps complet, en situation de cohabitation. Source : enquête Emploi du temps 2009-2010, Insee. 
Il y a donc bien un fort effet linéaire du salaire des femmes sur le temps qu'elles consacrent aux tâches domestiques, mais cet effet n'est pas le seul à l'œuvre. Les prédictions mises en évidence dans le graphique de droite de la figure II montrent en effet que la contrainte de positivité ne semble pas expliquer à elle seule le type de non-linéarité observée : la quantité de travail domestique ne tend pas asymptotiquement vers zéro, mais vers un niveau positif qui, même pour des niveaux de salaire élevés, reste supérieur à 2 heures. Il semble donc plutôt qu'il y ait un niveau incompressible de tâches qui reste effectué par les femmes, observé ici au niveau du volume global de tâches. Plusieurs hypothèses ont été formulées dans la littérature pour rendre compte de cette limite strictement supérieure à zéro. Killewald (2011) a mis en avant les contraintes économiques qui émergent dans le cas du recours à une forme d'externalisation des tâches, et en particulier les rendements décroissants liés aux coûts de transactions, de négociation ou de supervision de ce travail. D'autres types d'explications ont été mis en avant par la littérature sociologique : sans interpréter ces freins à la substitution en termes de «compensation» ou de "performance de genre », les études qualitatives

\section{Tableau 3}

\section{Estimation en régression linéaire pour le niveau agrégé}

\begin{tabular}{|c|c|c|c|c|c|c|}
\hline \multirow[b]{2}{*}{ Variable } & \multicolumn{2}{|c|}{$\begin{array}{l}\text { Modèle } 1 \\
\text { Femmes }\end{array}$} & \multicolumn{2}{|c|}{$\begin{array}{l}\text { Modèle } 2 \\
\text { Femmes }\end{array}$} & \multicolumn{2}{|c|}{$\begin{array}{l}\text { Modèle } 3 \\
\text { Femmes }\end{array}$} \\
\hline & Coefficient & Écart-type & Coefficient & Écart-type & Coefficient & Écart-type \\
\hline Part de salaire & $-235,47^{\star \star \star}$ & $(84,32)$ & $-103,94$ & $(102,78)$ & 25,81 & $(130,90)$ \\
\hline Part de salaire ${ }^{2}$ & $172,87^{\star \star \star}$ & $(81,08)$ & 82,36 & $(90,57)$ & $-22,95$ & $(110,18)$ \\
\hline Salaire de la femme & $-0,014^{\star \star}$ & $(0,0065)$ & $-0,046^{\star \star \star}$ & $(0,016)$ & - & - \\
\hline Salaire de la femme ${ }^{2}$ & - & - & $5,22 e-06^{\star \star}$ & $(2,34 \mathrm{e}-06)$ & - & - \\
\hline $1^{\text {er }}$ quartile (salaire) & - & - & - & - & $-0,088^{\star \star \star}$ & $(-0,030)$ \\
\hline $2^{\mathrm{e}}$ quartile & - & - & - & - & $-0,010$ & $(0,030)$ \\
\hline $3^{e}$ quartile & - & - & - & - & $-0,022$ & $(0,023)$ \\
\hline $4^{e}$ quartile & - & - & - & - & $-0,015^{*}$ & $(0,0078)$ \\
\hline Salaire de l'homme & $-0,0026$ & $(0,0034)$ & $7,5 e-05$ & $(0,0036)$ & 0,0019 & $(0,0038)$ \\
\hline Âge & $6,90^{\star \star}$ & $(2,31)$ & $7,30^{\star \star}$ & $(2,99)$ & $7,68^{\star \star}$ & $(3,01)$ \\
\hline$\hat{A} \mathrm{e}^{2}$ & $-0,058$ & 0,037 & $-0,060$ & $(0,0367)$ & $-0,066^{\star}$ & $(0,037)$ \\
\hline \multicolumn{7}{|l|}{ Diplôme le plus élevé : } \\
\hline Master & $-10,03$ & $(13,02)$ & $-2,21$ & $(13,47)$ & $-6,91$ & $(13,61)$ \\
\hline Licence & $-7,26$ & $(13,20)$ & $-0,470$ & $(13,53)$ & $-3,14$ & $(13,64)$ \\
\hline Baccalauréat & $-12,89$ & $(11,46)$ & $-10,06$ & $(11,52)$ & $-10,06$ & $(11,55)$ \\
\hline Brevet d'études & 2,91 & $(12,34)$ & 2,87 & $(12,32)$ & 3,03 & $(12,32)$ \\
\hline \multicolumn{7}{|l|}{ Un enfant (au moins) : } \\
\hline Âge $<3$ & $105,19^{\star \star \star}$ & $(9,42)$ & $103^{\star \star \star}$ & $(9,43)$ & $106,08^{\star \star \star}$ & $(9,45)$ \\
\hline $3 \leq$ âge $<6$ & $29,46^{\star \star \star}$ & $(7,57)$ & $29,52^{\star \star \star}$ & $(7,56)$ & $29,46^{\star \star \star}$ & $(7,56)$ \\
\hline Âge $<18$ & 25,15 & $(3,79)$ & $25,11^{\star \star \star}$ & $(3,79)$ & $25,05^{\star \star \star}$ & $(3,81)$ \\
\hline \multicolumn{7}{|l|}{ Zonage urbain } \\
\hline Grand pôle & $-18,38^{\star}$ & $(9,56)$ & $-16,17^{\star}$ & $(9,60)$ & $-16,16^{*}$ & $(9,61)$ \\
\hline Moyen pôle & $-21,87$ & $(13,67)$ & $-19,75$ & $(13,62)$ & $-19,68$ & $(13,64)$ \\
\hline Constante & 70,23 & $(61,60)$ & 43,62 & $(62,67)$ & 43,46 & $(63,61)$ \\
\hline$N$ & 1674 & - & 1674 & - & 1674 & - \\
\hline$R^{2}$ & 0,186 & - & 0,188 & - & 0,189 & - \\
\hline
\end{tabular}

Lecture : *** indique que le coefficient est significatif à $1 \%,{ }^{* *}$ à $5 \%$ et *à $10 \%$. On donne l'estimation des coefficients de régression pour chaque variable, avec entre parenthèses l'écart-type corrigé de l'hétéroscédasticité au niveau du cluster du ménage. Nous indiquons la significativité de ces coefficients à l'aide d'un test de Student, celle-ci est à interpréter à l'aide de la grille ci-dessus portant sur la p-value. L'interprétation de l'effet des variables indépendantes sur le temps de travail domestique se fait toutes choses égales par ailleurs : dans le modèle (1), 100 euros supplémentaires entraînent une baisse de 4 minutes et 36 secondes du temps consacré au ménage par les femmes de l'échantillon, en contrôlant pour l'effet de l'ensemble des variables indiquées dans la première colonne. Champ : couples hétérosexuels bi-actifs occupés, exerçant une activité salariée à temps complet, en situation de cohabitation. Source : enquête Emploi du temps 2009-2010, Insee. 
sur le sujet suggèrent en effet que les normes sociales ou identitaires continuent de peser fortement sur le choix domestiques des femmes (Lyonette et Crompton, 2014). Celles-ci, en particulier lorsqu'elles sont occupées à temps plein (Hochschild et Machung, 1989; Bereni et al., 2011 ; Baizàn et al., 2014), peuvent ressentir «qu'elles ne se consacrent pas assez à leur foyer », et ainsi le poids de leur déviance vis-à-vis d'un modèle normatif articulé autour de la figure du «breadwinner» (Acker, 1988; Delphy et Leonard, 1992), qui continue d'attribuer la responsabilité du travail domestique en priorité aux femmes. Nous ne tranchons pas ici sur le sens donné à cette inflexion, nous souhaitons simplement insister sur le fait qu'une relation non linéaire ne signifie pas nécessairement qu'il y ait un retournement de la courbe. Les normes de genre jouent de manière complexe, en dehors du cas extrême où celles-ci inversent la relation entre ressources économiques et temps de travail domestique, ce qu'ont bien mis en évidence Lyonette et Crompton (2014) dans le cas du Royaume-Uni.

Nos estimations montrent ainsi que les femmes salariées à temps plein cumulent une activité marchande et un niveau élevé de tâches domestiques, ce qui souligne les difficultés possibles liées à l'«articulation» (Ollier-Malaterre, 2008) d'un emploi à niveau de rémunération élevée et de la vie familiale (Acker, 2009), et ceci indépendamment des revenus du conjoint ou de la négociation intra-ménage telle que nous l'avons mesurée. Ce schéma est donc également en cela différent de l'interprétation en termes de «NDG », qui est centrée autour du processus de négociation au sein du couple, et donc autour du mari comme «spectateur» de cette «performance». Ce résultat portant sur l'effet indépendant du salaire des femmes va dans le sens d'une remise en cause des approches qui placent la négociation au cœur de l'analyse de l'organisation de la vie domestique : l'effet non significatif, à la fois linéaire et quadratique, de la part de salaire dans les modèles (2) et (3) plaide en faveur de l'approche «absolue». De plus, le coefficient de corrélation entre le salaire et la part de salaire est de 0,53 pour les femmes de notre échantillon: ainsi, les femmes qui gagnent plus que leur mari sont aussi celles qui sont les mieux rémunérées dans l'absolu; néanmoins ces deux variables ne sont pas corrélées au point qu'on ne puisse pas départager entre les approches «absolue» et "relative». Il y a bien une possibilité d'observer des effets autonomes de ces deux variables, or, à niveau de salaire équivalent, nous n'observons aucun effet des ressources relatives des femmes sur la quantité de travail domestique qu'elles effectuent.

\section{L'effet des autres variables caractéristiques}

Qu'en est-il de l'effet des autres variables? Le fait d'avoir des enfants, en particulier en bas âge, augmente mécaniquement la quantité de travail domestique des femmes (modèle (1)) : une heure et 45 minutes de plus par semaine pour un enfant de moins de 3 ans, cet effet étant décroissant avec l'âge des enfants, en particulier avec l'entrée en maternelle après l'âge de trois ans. Le modèle (4), très proche du modèle (1), montre que l'effet d'un enfant de moins de 3 ans supplémentaire est deux fois plus important sur le travail domestique des femmes que sur celui des hommes (104 minutes dans un cas contre 57 dans l'autre), accroissant les inégalités au sein des couples (voir Régnier-Loilier (2009) et Sullivan et Gershuny (2013) pour des prédictions similaires). Le fait de résider au sein ou en périphérie d'un grand pôle urbain diminue la quantité de travail domestique effectuée par les femmes (modèle (1)) de 16 minutes hebdomadaires, mais cet effet n'est significatif que dans les premiers modèles (1), (2) et (3). Le niveau de diplôme n'est pas significativement corrélé à la quantité totale de travail domestique réalisée par les femmes ${ }^{34}$.

Plutôt que de s'interroger sur les raisons idiosyncratiques qui poussent les femmes à ne pas transformer linéairement plus de rémunérations en moins de tâches domestiques, nous allons tenter de décrire la processus de substitution en s'interrogeant d'une part aux mécanismes spécifiques à certaines tâches domestiques, et d'autre part à ce que deviennent ces tâches non prises en charge par les femmes.

\section{Une « relève » des hommes ?}

$\mathbf{L}$ es femmes font donc moins de travail domestique à mesure que leurs revenus augmentent, même si en moyenne elles continuent à y consacrer plus de temps que leur conjoint. La question qui nous intéresse est alors celle-ci : pourquoi la substitution de l'homme à la femme pour certaines tâches domestiques stagne-t-elle pour des revenus élevés?

34. Sauf dans le cas du linge pour les modèles (7) et (7bis), nous y reviendrons. 
Schématiquement: si les femmes à revenus élevés font moins de travail domestique que celles à revenus plus faibles, elles pourraient en prendre encore moins à leur charge. Nous nous intéresserons dans un premier temps à la relation entre le salaire de la femme et le travail domestique du conjoint. Les résultats précédents suggèrent qu'il y aurait un volume fini de tâches que les femmes peuvent déléguer, et que leur capacité à transférer ces tâches diminue à mesure que leur salaire augmente. Le niveau de travail domestique incompressible serait atteint de cette façon. Si nous observons un effet non linéaire symétrique du salaire des femmes sur le volume de tâches effectué par les hommes, nous pourrons affirmer qu'au moins une partie de cette "incompressibilité » est due au fait que les femmes parviennent à déléguer aux hommes un certain volume de tâche mais que ce volume délégable est progressivement épuisé à mesure que leur salaire augmente. Il s'agit donc d'une part de savoir si la non-linéarité observée précédemment s'explique par un effet non linéaire symétrique sur le travail domestique des hommes, et d'autre part de savoir si ces effets sont de niveau équivalents, ou si le niveau total de tâches effectué par les conjoints varie. Cette question de la substituabilité des tâches de l'homme et de la femme en fonction de modifications des ressources économiques a été introduite par Thibout $(2014)^{35}$, qui cherche à modéliser précisément ces mécanismes à partir d'une fonction de production domestique et des niveaux de salaire relatifs. Aboutir à des hypothèses structurelles testables à partir de modèles de choix, comme elle le montre, s'avère compliqué, et notre perspective aura avant tout un but descriptif de comparaison des effets différentiels du salaire des femmes sur le temps de travail domestique des conjoints.

Dans cette optique, nous utiliserons une version modifiée du modèle (2) ci-dessus, en excluant les variables liées au pouvoir de négociation (part de salaire et part de salaire quadratique) : la partie précédente ayant permis de remettre en cause le potentiel explicatif de ces variables, nous les éliminons ici dans un souci de parcimonie $^{36}$. Nous adoptons donc une approche « absolue » en ne conservant comme variables explicatives que le salaire de la femme et le salaire quadratique de la femme ${ }^{37}$. Pour les hommes, nous faisons le choix de ne prendre en compte que le salaire comme variable de contrôle, et non une mesure quadratique de ce salaire, car ce qui nous intéresse ici avant tout n'est pas tant de modéliser les choix des hommes que de comprendre l'effet sur les hommes des modifications des ressources économiques de leur conjointe.

Nous estimons séparément ce modèle (4) pour les femmes et les hommes, les résultats sont indiqués dans les deux premières colonnes du tableau $4^{38}$. Il y a un effet négatif significatif du salaire des femmes sur le temps consacré par les hommes aux tâches domestiques. D'autre part, l'effet quadratique du salaire de la femme est non-significatif : il n'y a pas de non-linéarité de la relation entre salaire de la femme et travail domestique de l'homme. La relève partielle des hommes pour le volume global de tâches ne semble donc un pas facteur explicatif de la non-linéarité observée dans le cas des femmes. Nous ne pouvons comparer les effets différents entre hommes et femmes dans le modèle (4) que pour un niveau de salaire fixé, puisque dans le cas des femmes la relation estimée est quadratique. Pour un niveau de salaire médian de 1500 euros, l'effet du coefficient estimé dans le modèle (4) est de - 0,046 pour les femmes : cent euros de plus équivaut à 4 minutes 36 secondes de travail domestique en moins. Ainsi, pour ce niveau médian, l'effet du salaire des femmes sur leur quantité de travail domestique est plus de quatre fois supérieur $(-0,046 / 0,010)$ à l'effet sur le volume de tâche effectué par les hommes ${ }^{39}$. Il ne s'agit pas d'un jeu à somme nulle ${ }^{40}$, s'il y a une relève des hommes, celle-ci ne semble que très partielle : une augmentation de 100 euros du salaire des femmes entraîne une hausse d'une minute de la quantité de travail domestique effectuée par les hommes. De ce point de vue, nous confirmons les résultats mis en évidence par Thibout (2014). D'autre part, le pouvoir explicatif du modèle (4) est largement plus faible dans

35. Voir en particulier le chapitre 2 écrit avec la collaboration de Catherine Sofer.

36. Nous nous intéressons en effet ici plus spécifiquement aux effets autonomes des ressources économiques des femmes sur le comportement de conjoints. Les modèles (4)-(8) n'ont pas pour objectif de modéliser le mieux possible la répartition du travail domestique, mais de comparer les effets globaux, et par tâches, différents pour les hommes et les femmes.

37. Nous avons estimé une version différente des modèles (4)-(8) en incluant la part de salaire des femmes de degré 1 et 2, testant ainsi la présence d'un effet du pouvoir de négociation des femmes sur le travail domestique des hommes, malgré l'effet non significatif de ces variables sur le travail domestique des femmes. Les résultats diffèrent peu de ceux présentés ici dans le tableau 4. 38. Ce tableau résume les résultats des modèles (4)-(8) et (4bis)-(7bis) en indiquant le poste considéré, ce qui indique que la variable dépendante est le nombre de minutes consacré à la tâche en colonne.

39. Le modèle (3) nous a permis de mettre en évidence que l'effet du salaire des femmes était plus fort pour le premier quartile de salaire, et nettement plus faible pour le 4ème quartile. Cet effet mesuré par le modèle (4) est de - 0,048 pour le $25^{e}$ percentile de salaire, et de - 0,043 pour le $75^{e}$ percentile de salaire. 40. On constate également que la valeur du coefficient pour la variable "salaire " ne change pas significativement entre les modèles (2) et (4), lorsqu'on exclut les mesures des ressources économiques relatives. 


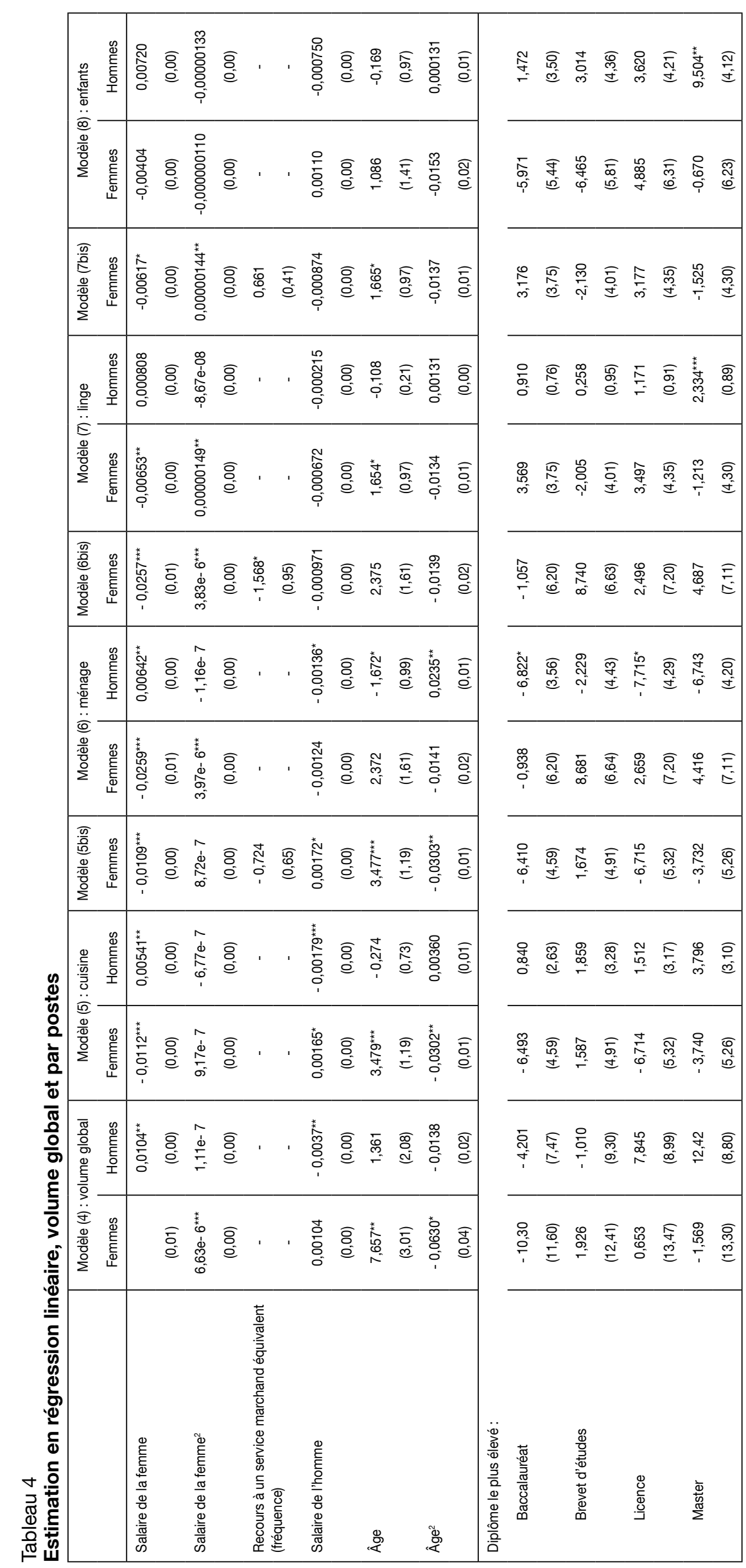




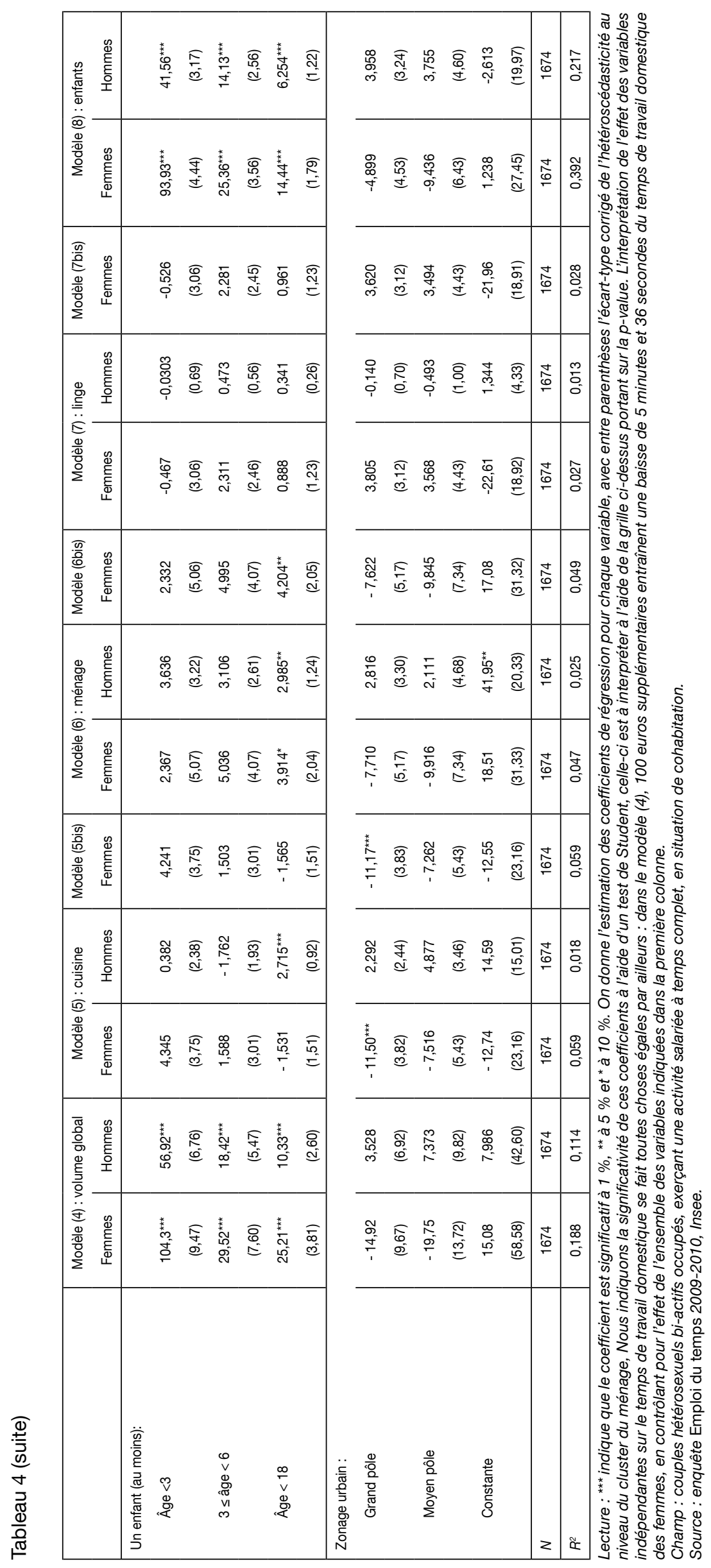


le cas des hommes: le $R^{2}$ de 0,114 souligne que le salaire des femmes explique une part plus faible de la variance du travail domestique effectué par les hommes. Les hommes ne prennent pas à leur charge l'ensemble des tâches délaissées par les femmes ${ }^{41}$, et l'effet d'une augmentation du salaire des femmes est constant : il ne varie pas selon la valeur du salaire à laquelle on l'observe.

En dehors du faible volume de tâches pris en charge par les hommes lorsque le salaire des femmes augmente, consacrer moins de temps aux activités domestiques pour les femmes peut être, à mesure que leur revenu augmente, synonyme soit d'externalisation sur le marché, soit de suppression pure et simple des tâches, ou d'une diminution de la qualité des produits de ce travail - question sur laquelle nous reviendrons plus loin. Nous allons nous intéresser à présent aux différentes tâches que ce volume global recouvre, afin d'identifier les tâches qui expliquent d'une part la relation négative entre le salaire des femmes et le volume global de travail consacré à différentes tâches domestiques, et d'autre part si certaines d'entre elles présentent le même type de non-linéarité que pour le volume global (1).

\section{Quels effets pour quelles tâches?}

$\mathbf{L}$ es revenus du travail des femmes ont bien un effet sur la quantité globale de travail domestique qu'elles effectuent, mais par quelles tâches spécifiques s'opère cette diminution? Nous avons divisé notre agrégat en quatre postes spécifiques: cuisine, ménage, linge et temps consacré aux enfants (voir le tableau 2 pour une liste précise des tâches), et évalué le modèle (4) pour chacun des quatre postes (respectivement modèles (5), (6), (7) et (8)), en estimant à chaque fois le modèle pour les hommes et les femmes. Nous pensons en effet qu'en rester à une mesure globale du travail domestique empêche d'identifier les logiques propres à chaque tâche, et, plus généralement, de s'interroger sur la validité de l'agrégation, étant données la diversité et les spécificités potentielles des tâches que ces agrégats recouvrent.

\section{L'ajustement du travail domestique des femmes porte plus sur le ménage et le linge que sur la cuisine...}

Ces estimations montrent que les femmes ajustent leur temps de travail domestique par les postes identifiés comme le cœur du travail ménager : la cuisine (5), le ménage (6) et le linge (7). Un résultat similaire a déjà été mis en évidence dans le cas des femmes françaises subissant une période de chômage par Solaz (2005). Avant d'entrer dans le détail des coefficients, il convient de mettre en avant la faiblesse des $R^{2}$ dans les modèles (5), (6) et (7), nous ne parvenons à expliquer qu'une part très faible de la variance totale du temps consacré par les femmes au ménage, au linge et à la cuisine. Le pouvoir explicatif des modèles par type de tâches est en particulier bien plus faible que pour le volume global.

La figure III représente les prédictions des effets marginaux conditionnels significatifs pour les modèles (4) à (7). On observe une relation négative et non linéaire dans le cas du linge et de la cuisine; les coefficients de régression du salaire et du salaire quadratiques étant significatifs dans les deux cas. Le ménage est le poste pour lequel l'effet du salaire est le plus fort: pour un niveau médian de revenu, une augmentation de 100 euros de salaire entraîne une baisse de 2 minutes du temps consacré au ménage. Pour le linge, l'effet est très faible puisqu'à un niveau de revenu médian, ce modèle prédit une baisse de 24 secondes pour une augmentation de 100 euros du salaire de la femme ${ }^{42}$.

Le modèle (5) identifie une relation linéaire entre le niveau de salaire et le temps passé à faire la cuisine, puisque le coefficient devant le terme quadratique n'est pas significatif. Il semble donc que la non-linéarité observée dans le cas du modèle (4) soit avant tout due aux tâches liées au ménage - dans une moindre mesure au linge - et non à la cuisine. Une augmentation de 100 euros de salaire entraîne une baisse de 1 minute et 7 secondes du temps passé à faire la cuisine. Même si l'on corrige ces effets du fait que le temps moyen consacré à ces tâches diffère pour les femmes (cf. tableau 1), on remarque que l'impact d'une augmentation de salaire de la femme est, même relativement, nettement plus fort dans le cas du ménage que dans le cas de la cuisine. Intéressons nous de

41. Pour une analyse qualitative des implications possibles de cette "relève » partielle, voir en particulier le chapitre 6 du livre d'Hochschild et Machung (1989). Les auteures montrent les différentes stratégies de compensations utilisées par les hommes, comme le soutien émotionnel, qui par moment peut remplacer ou se substituer à un investissement en temps réclamé par la femme. 42. Dans le cas du ménage, le retournement de la courbe estimée est extérieur au domaine d'estimation puisque le modèle prédit une relation positive entre salaire et temps consacré au ménage pour une seule observation. Dans le cas du linge, la concavité est nettement plus forte, mais le modèle prédit un retournement de la courbe uniquement pour 17 femmes de l'échantillon. 
plus près à cette différence de forme fonctionnelle: on peut émettre l'hypothèse que le ménage et le linge recouvrent un ensemble d'activités davantage considérées comme des corvées et ainsi plus rapidement supprimées de l'agenda des femmes (Sullivan et Gershuny, 2013), la contrainte consistant à maintenir un niveau minimal positif de tâches s'activant ainsi plus fortement. La cuisine est à l'inverse une activité que les femmes suppriment moins rapidement de leur agenda; la substitution est plus lisse pour cette activité et l'effet ne varie pas en fonction du niveau de salaire. Un élément à l'appui de cette hypothèse: la base Emploi du temps comporte une variable qui renseigne sur la pénibilité d'une tâche, évaluée subjectivement, bien qu'il existe un fort biais de sélection puisque seuls les individus ayant effectué ces tâches durant la semaine d'enquête y sont renseignés. Malgré cela, si l'on compare les ressentis exprimés par les femmes quant à la cuisine et au ménage, on remarque qu'elles sont $14 \%$ à trouver que faire la cuisine est une corvée, contre $25 \%$ qui trouvent que c'est une activité agréable. Pour le ménage, elles ne sont que $4 \%$ à trouver cela agréable, contre $43 \%$ à considérer cette tâche comme une corvée ${ }^{43}$. Ce résultat est proche d'éléments mis en évidence dans le cas de la France par Hook (2010) et des Etats-Unis par Killewald et Gough (2010).

\section{...et ne concerne pas le temps passé à s'occuper des enfants}

À l'inverse, le temps passé à s'occuper des enfants n'est pas une variable d'ajustement (8), il ne varie pas en fonction des évolutions du salaire des femmes ${ }^{44}$. Ce résultat, qui démarque le temps passé à s'occuper des enfants du reste des tâches domestiques, a déjà été mis en évidence dans le cas de l'Italie par Bloemen et

43. Malgré l'échantillon réduit qui permis le calcul de ces moyennes, nous avons effectué un test de Student qui montre qu'elles diffèrent bien significativement. II aurait également été intéressant de voir si cette évaluation modifie la pente de la courbe estimée pour différentes catégories de femmes ayant différentes évaluation des tâches, néanmoins le biais de sélection mentionné ci-dessus nous empêche de pousser davantage l'analyse à ce stade.

44. On peut cependant remarquer que l'effet lié à la présence d'un enfant, en particulier en bas âge dans le couple, est plus de deux fois plus fort dans le cas des femmes : il augmente la quantité de travail domestique effectué par la femme de 93 minutes.

\section{Figure III}

Prédictions par types de tâches (modèles 4-7)

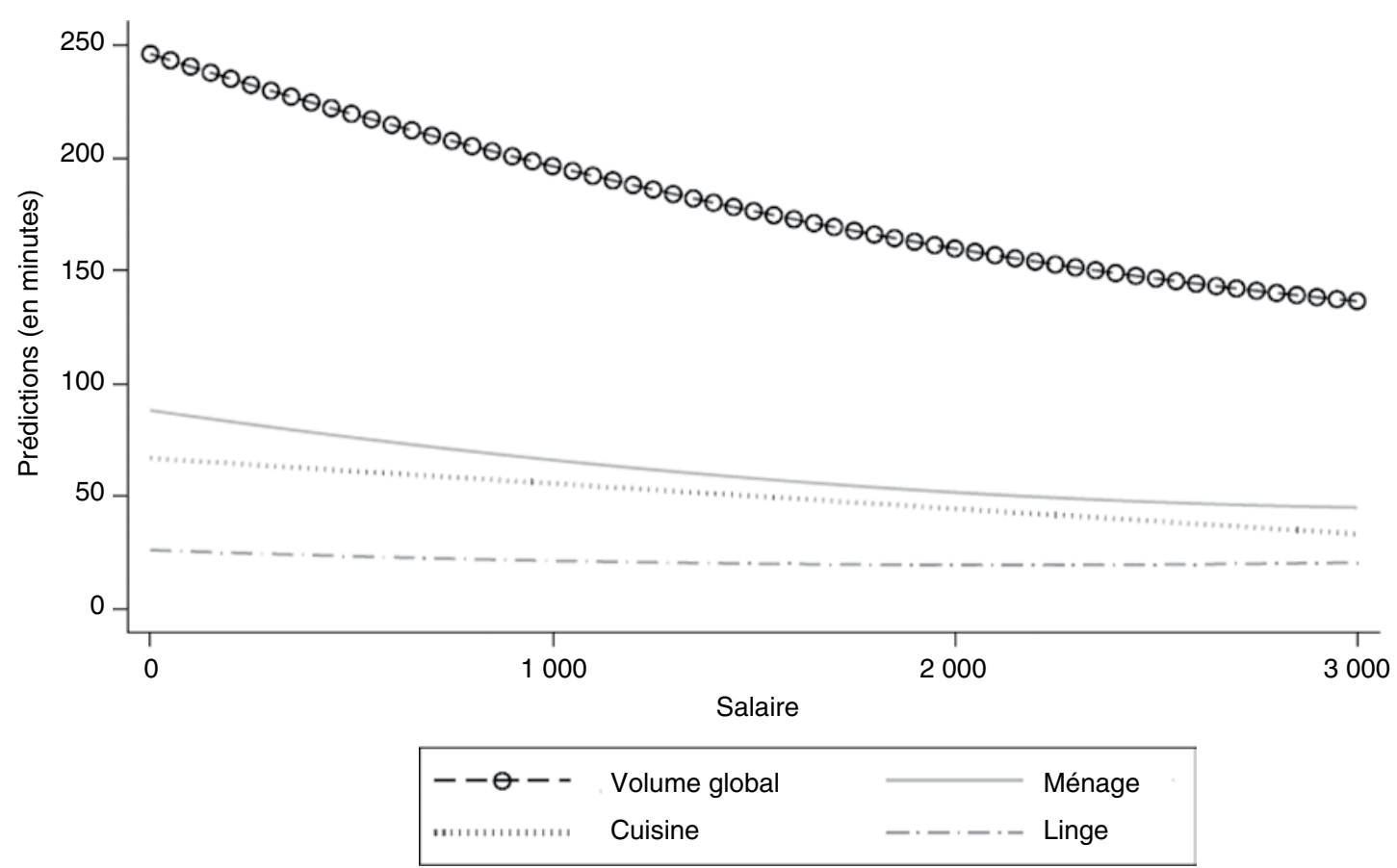

Lecture : ces courbes représentent les effets marginaux conditionnels prédits par les modèles (4), (5), (6) et (7). Les paramètres estimés sont les constantes mesurées dans le tableau 4, corrigées des moyennes conditionnelles des variables du modèle. Pour les modèles (4) et (6) et (7), les effets marginaux sont obtenus en combinant les coefficients de régression linéaires et quadratiques de la part de salaire et du salaire respectivement. Nous ne représentons que la forme linéaire pour le modèle (5).

Champ : couples hétérosexuels bi-actifs occupés, exerçant une activité salariée à temps complet, en situation de cohabitation. Source : enquête Emploi du temps 2009-2010, Insee. 
Stancanelli (2008). Baizàn et al. (2014), dans une étude portant sur cinq pays européens dont la France, ont notamment pu montrer que le choix du temps consacré aux enfants est davantage dû à l'origine sociale qu'aux ressources économiques des couples, un résultat similaire à celui mis en évidence par Guryan et al. (2008). Similairement, Pailhé et Solaz (2008) ont identifié un effet très faible du chômage des conjoints sur le changement de la répartition des charges familiales liées aux enfants, ceux-ci restent réticents à déléguer ces tâches malgré les variations des disponibilités temporelles (voir également Hook et Wolfe (2013) pour une étude sur d'autres pays $)^{45}$. La littérature qualitative a pu mettre en évidence des résultats similaires: Bereni et al. (2011) montrent que pour les femmes de la haute fonction publique française, la conciliation de charges de famille et d'une activité professionnelle exigeante nécessite des « acrobaties physiques et mentales » pour maintenir une forme d'investissement dans la prise en charge et l'éducation des enfants ; une « charge mentale » (Bereni et al., 2011) qui pèse moins sur les autres postes du travail domestique. On peut enfin supposer que cette absence d'effet est en partie due à la restriction de notre échantillon aux couples bi-actifs occupés à temps plein. En effet, ce sont des couples pour qui le choix du temps partiel ne s'est pas imposé comme une solution, or ce sont souvent les femmes qui subissent ce choix. Les femmes retenues ici ont semble-t-il pu mettre en place des arrangements nécessaires à la conciliation d'une prise en charge des enfants avec une activité à plein temps exercée par les deux conjoints, la question des ressources économiques devenant dans ce cas moins primordiale pour elles.

\section{Les hommes prendraient davantage en charge les tâches délaissées par les femmes dans le cas de la cuisine que du ménage}

Qu'en est-il du comportement des hommes vis-à-vis de ces tâches? En particulier, les variations des ressources économiques des femmes ont-elles des effets différents sur le temps des hommes suivant le poste considéré ?

Pour le ménage et la cuisine, on observe une relation linéaire significative (cf. tableau 4), et le coefficient devant le terme quadratique est non significatif. En cela, ces résultats sont cohérents avec les estimations portant sur le volume global (modèle 4). Dans le cas du ménage (modèle 6), la non-linéarité ne semble pas due au fait que les hommes réagissent de manière non linéaire aux augmentations de salaire de la femme. En reprenant les résultats de la section précédente, nous pouvons comparer l'impact d'une augmentation de cent euros du salaire de la femme, pour un niveau de revenu médian : il est de 2 minutes dans le cas des femmes, et de 39 secondes dans le cas des hommes ${ }^{46}$. Le salaire de la femme a un effet différent sur le temps de travail domestique de la femme et sur celui de l'homme : seule une fraction de ce temps est reprise à leur compte par les hommes. Pour la cuisine, l'estimation du modèle (5) montre qu'une augmentation de salaire de 100 euros des femmes entraîne une augmentation du temps consacré par les hommes à cette tâche de 32 secondes, contre une diminution de 1 minute et 7 secondes dans le cas des femmes. Les hommes consacrent, comme les femmes, en moyenne plus de temps au ménage qu'à la cuisine (tableau 1), mais ce résultat montre que, contrairement aux femmes, l'effet du salaire des femmes sur le temps consacré au ménage et sur celui passé à faire la cuisine est quasiment le même ( 32 secondes contre 39 secondes) pour les hommes. Cet effet différentiel entre tâches est notoirement plus faible que celui observé pour les femmes, qui, lui, est proche d'un rapport du simple au double ( 2 minutes contre 1 minute et 7 secondes).

Les moyennes du tableau 1 ne montraient pas de particularité spécifique au ménage ou à la cuisine : pour ce qui est de la différence homme/ femme, les écarts varient dans des proportions équivalentes pour les deux tâches (les femmes consacrent similairement $153 \%$ plus de temps que les hommes à ces deux tâches). Or l'estimation des modèles (5) et (6) pour les hommes montre que le l'effet différentiel entre la cuisine et le ménage est très différent entre les hommes et les femmes. Cet écart des coefficients entre

45. Ces résultats montrent que les déterminants du travail domestique liés à la prise en charge des enfants semblent différents des autres types de tâches. En conséquence, nous avons estimé les modèles (1), (2) et (3) en excluant le temps consacré à s'occuper des enfants. Sans surprise, les variables liées au nombre et à l'âge des enfants ont dans ce cas un effet non significatif sur le travail domestique. Pour ce qui est des autres variables dépendantes, les coefficients et les écarts-types changent peu, seul l'effet du salaire de la femme pour le quatrième quartile devient non significatif dans l'estimation $d u$ modèle (3). Un changement important concerne le $R^{2}$ qui passe de 0,186 à 0,083 pour le modèle (1) et évolue dans des proportions similaires pour les modèles (2) et (3). Ceci met en évidence le fait qu'une part important de la variance du travail domestique totale capturée par notre spécification provient de la corrélation entre le nombre et l'âge des enfants et la part du temps de travail domestique dédiée aux enfants. Les déterminants de la prise en charge des enfants étant semble-t-il différents, il n'est pas surprenant d'observer des estimations similaires de la relation entre travail domestique et ressources économiques, malgré une variance totale expliquée plus faible.

46. En gardant à l'esprit que l'effet est linéaire dans le cas des hommes. 
les hommes et les femmes montre que l'augmentation du salaire des femmes a un effet relativement plus fort sur le temps consacré à la cuisine vis-à-vis du temps consacré au ménage pour les hommes, même si cet effet reste faible. Les femmes se déchargent davantage des tâches liées au ménage que de celles de la cuisine, mais cela ne se traduit pas par une prise en charge plus forte du ménage vis-à-vis de la cuisine par les hommes; ceci confirme un résultat mis en évidence par Hook (2010). En réalité, ce raisonnement s'étend au linge puisqu'on n'observe aucun effet significatif du salaire des femmes dans ce cas. Enfin, le modèle (8) montre que, comme pour les femmes, le temps consacré par les hommes à s'occuper des enfants est, lui aussi, insensible aux variations de salaire des femmes.

De même que pour le volume global, on n'observe pas de non-linéarité pour les hommes lorsqu'on analyse les logiques spécifiques aux quatre postes identifiés. Néanmoins, et même si l'effet mesuré est faible, il semble qu'il y ait une propension plus grande des hommes à prendre en charge le temps délaissé par les femmes dans le cas de la cuisine que dans celui du ménage.

\section{L'externalisation des tâches et la relation salaire-temps de travail domestique}

S i les hommes ne prennent que partielle$\bigcirc$ ment en charge les tâches délaissées par le femmes, que devient ce temps de production domestique ? Le recours à des services extérieurs marchands est l'un des mécanismes principaux mis en avant (Gupta, 2007 ; Gupta et Ash, 2008) pour expliquer l'impact négatif d'augmentations du salaire des femmes sur le temps consacré aux travaux domestiques, ce que Killewald (2011) nomme la stratégie du «buying out ».

\section{Le recours à des services extérieurs est de faible ampleur et ne concerne que peu de ménages,...}

Le tableau 5 présente des données relatives au recours à ces services extérieurs. Nous indiquons pour chaque poste la proportion de couples qui utilisent une aide extérieure rémunérée dans la semaine, et les quartiles de fréquence (en volume) de recours parmi les couples ayant eu recours au moins une fois à une aide extérieure. On peut noter que les ménages ont en moyenne assez peu recours à ces services, en particulier pour le ménage, et, parmi ceux qui y ont recours, cela reste assez occasionnel. Seuls $11,35 \%$ des ménages ont recours à une aide extérieure pour le ménage, et la médiane se situe à 2 heures par semaine parmi ces couples. Le domaine pour lequel le recours semble le plus courant - ou en tout cas celui pour lequel le substitut marchand à la tâche est le mieux mesuré - est celui du recours à un service de garde rémunéré, or il s'agit aussi de la tâche la moins corrélée aux évolutions de revenus. Nous indiquons également le salaire moyen des femmes ayant recours à ces services et le percentile de revenus auquel ce salaire correspond. Les femmes

Tableau 5

Statistiques descriptives, recours à l'externalisation

\begin{tabular}{|l|c|c|c|c|}
\hline & $\begin{array}{c}\text { Ménage : } \\
\text { aide extérieure } \\
\text { rémunérée }\end{array}$ & $\begin{array}{c}\text { Linge : } \\
\text { recours } \\
\text { au pressing }\end{array}$ & $\begin{array}{c}\text { Cuisine : } \\
\text { commande et livraison } \\
\text { de plats préparés }\end{array}$ & $\begin{array}{c}\text { Enfants : } \\
\text { service de garde } \\
\text { rémunérée }\end{array}$ \\
\hline Pourcentages de couples & 11,35 & 37,63 & 21,98 & 23,48 \\
\hline Type de mesure & $\begin{array}{c}\text { Nombre d'heures/ } \\
\text { semaine }\end{array}$ & Fréquence & Fréquence & $\begin{array}{c}\text { Nombre d'heures/ } \\
\text { semaine }\end{array}$ \\
\hline Parmi ceux ayant recours & 1 & 1 & & 7 \\
Quartile 1: & 2 & 1 & 1 & 19 \\
Quartile 2: & 3 & 3 & 1 & 35 \\
Quartile 3: & 2500 & 1865 & 1746 & 1730 \\
Salaire moyen : & 90 & 66 & 61 & 61 \\
Percentile de salaire correspondant & & & & \\
\end{tabular}

Lecture : les quartiles et les percentiles indiqués portent uniquement sur les couples ayant recours au moins une fois ou une heure à un service extérieur durant la semaine de l'enquête. Ils indiquent les quartiles et les percentiles de salaire mensuel de la femme au sein du couple considéré. Par exemple, le salaire moyen des femmes ayant recours au moins une fois au pressing durant la semaine de l'enquête est de 1865 euros mensuels, ce qui correspond au 66 percentile de salaire pour l'ensemble des femmes de l'échantillion.

Champ : couples hétérosexuels bi-actifs occupés, exerçant une activité salariée à temps complet, en situation de cohabitation.

Source : enquête Emploi du temps 2009-2010, Insee. 
appartenant à des ménages ayant recours à un service d'aide au ménage font partie des plus riches de l'échantillon, avec un salaire moyen correspondant au neuvième décile de revenus. Ce tableau suggère déjà que les mécanismes d'externalisation concernent une fraction limitée de la population, et sont dans l'ensemble de faible ampleur.

\section{... sa mesure, ainsi celle que de ses effets, est encore difficile et imparfaite}

Nous allons ici chercher à savoir quelle part de la corrélation négative et (le cas échéant suivant la tâche) non linéaire identifiée par les modèles précédents peut s'expliquer par un recours plus important des femmes à des services marchands extérieurs. Nous cherchons donc moins à savoir si les femmes ont recours, et dans quelle mesure, à des services marchands lorsque leur salaire augmente, mais de savoir à quel point cette association explique ou non le signe et la forme fonctionnelle identifiée précédemment. Cependant, plusieurs problèmes liés à l'identification de ces effets doivent d'abord être mentionnés.

En premier lieu, la relation apparente entre la fréquence de recours aux services extérieurs et le temps de travail domestique des femmes peut être faussée car les deux variables ont des déterminants communs, en particulier le salaire. Il se peut en effet d'une part qu'une augmentation du recours à des services extérieurs ne se traduise pas par une diminution de la quantité de travail domestique effectuée par la femme. La fréquence du recours au pressing est, par exemple, souvent utilisée comme une mesure du recours à un service marchand équivalent à « s'occuper du linge », or la corrélation positive éventuelle entre « recours au pressing » et « salaire » peut simplement être due à des achats de biens vestimentaires différents qui nécessitent ce type de traitement. Il en est de même pour la fréquence du recours à une aide extérieure pour le ménage : une femme ayant des revenus élevés et vivant dans un vaste logement peut avoir recours à une aide ménagère complémentaire occasionnelle tout en consacrant autant de temps à faire le ménage qu'une femme qui a des revenus moins élevés mais une surface moins grande à entretenir ${ }^{47}$.La surface du logement ne faisant pas partie de l'enquête, nous ne pouvons pas distinguer entre ces deux situations ${ }^{48}$. Pour ce qui du temps consacré aux enfants, nos données indiquent dans la même optique un effet positif significatif ${ }^{49}$ du salaire des femmes sur le nombre d'heures au cours desquelles le ménage à recours à un système de garde rémunérée, alors même que l'estimation du modèle (8) montre que le salaire des femmes n'a pas d'influence sur le temps qu'elles consacrent à s'occuper des enfants ${ }^{50}$. Ceci indique donc que, pour identifier correctement une substitution entre tâches en interne et tâches en externe, il faut pouvoir estimer les effets pour des « structures » de productions domestiques équivalentes ${ }^{51}$, sinon on s'expose à une mauvaise interprétation des phénomènes observés. Sullivan et Gershuny (2013) introduisent en particulier l'idée d'une « causalité inverse » : ce sont les femmes qui effectuent le plus de travail domestique qui ont également le plus souvent recours à l'externalisation, et non l'inverse. L'identification d'un effet de substitution entre production interne et externe s'avère difficile et c'est pourquoi nous avons décidé de nous concentrer sur la mesure de la part de l'association entre le salaire des femmes et leur temps de travail domestique qui est due à l'achat de services marchands extérieurs ; sans chercher à démêler les mécanismes causaux complexes liés à l'externalisation en général.

Une deuxième difficulté est liée au fait qu'on peut observer un impact du recours à des services marchands sur le travail domestique des femmes sans que celui-ci soit dû à une augmentation de salaire. Un ménage peut modifier ses attentes en termes de tâches domestiques ou diminuer la valeur qu'il attribue au fait que celles-ci soient effectuées en interne, sans que cela soit dû à des revenus plus élevés. En particulier, modéliser l'association entre le recours aux services extérieurs et le temps de travail domestique de la tâche équivalente ne nous dit a priori rien sur le fait que celle-ci est due à une variation de salaire.

47. Cette affirmation n'a rien de normatif quant au fait que ce sont les femmes qui doivent gérer ce type de problèmes. En général, elle vise simplement à illustrer une situation théorique ou deux femmes salariées consacrent le même volume de temps aux tâches ménagères, malgré des niveaux de salaire et de recours à des services marchands très différents.

48. Contrairement à Killewald (2011) qui possède cette information.

49. Nous avons simplement régressé la fréquence du recours à une garde rémunérée sur le salaire de la femme pour le groupe des femmes et le test de Student sur le coefficient de régression montre que l'effet est significatif avec une $p$-value inférieure à 0,01 .

50. Par ailleurs, nous nous ne nous intéresserons pas au temps consacré aux enfants dans cette partie pour cette raison.

51. En économie des organisations, la même question se pose lorsqu'on s'intéresse à l'out-sourcing. Il faut raisonner sur des entreprises qui ont une organisation similaire de la production, aussi bien au niveau de l'échelle de celle-ci que de la technologie requise. 
Un troisième type de limite concerne l'identification et la mesure de variables qui permettent une approximation du recours à des substituts marchands pour les tâches ménagères effectuées en interne. L'enquête Emploi du temps contient des variables spécifiques à certaines tâches qui permettent de savoir si un ménage a eu recours à des types de services extérieurs et à quelle fréquence. Ces variables ne font pas partie des carnets journaliers pour la simple raison que ceux-ci renseignent les activités effectivement réalisées par les individus, et non l'ensemble des tâches ménagères faites par un membre du ménage ou une personne extérieure à celui-ci. Elles présentent de plus de nombreuses limites qui, selon nous, ne permettent pas à ce stade de mesurer finement le recours des ménages à l'externalisation des tâches ménagères. Pour ce qui est du ménage, nous pouvons savoir combien de fois le ménage a eu recours à un service extérieur mais nous ne connaissons, ni le prix, ni la durée du recours au service, ni la surface du logement du couple. Dans le cas de la cuisine, la seule proxy permettant de capturer ce recours aux services extérieurs est une variable qui indique le nombre de fois où le ménage a eu recours « à la commande et à la livraison de plats préparés ». Il s'agit selon nous d'une faible approximation des substituts possibles à la préparation de la cuisine, puisque le fait d'aller au restaurant ou d'acheter des plats préparés, entre autres, ne sont pas pris en compte. De la même manière, le recours au pressing ne recouvre qu'une partie des tâches liées au linge ; il ne concerne que les vêtements nécessitant un tel traitement.

\section{Une externalisation faible et pour un petit nombre de femmes}

Nous donnons néanmoins en annexe 2 les résultats de trois régressions logistiques (cf. tableau 7) qui estiment la probabilité d'avoir recours à un service extérieur en fonction du salaire de la femme. Nous reportons également trois régressions linéaires complémentaires, en restreignant l'échantillon aux femmes ayant recours à une aide extérieure, avec le but de mesurer, parmi ces femmes, l'impact d'augmentations de salaires sur le volume d'externalisation des tâches. Nous utilisons ici l'approche suggérée par Sullivan et Gershuny (2013), en conservant les mêmes variables de contrôle que dans les modèles précédents. Le pouvoir explicatif de ces modèles s'avère fort pour ce qui est du ménage et de la garde d'enfants. Pour le ménage, la régression logistique explique $25 \%$ de la variance totale de la probabilité d'avoir recours à une aide extérieure; pour la garde d'enfant, la part expliquée est de $37 \%$. Les régressions linéaires portant sur le volume de recours présentent un $R^{2}$ beaucoup plus faible dans le cas du ménage $(0,11)$, et similaire dans le cas de la garde d'enfant $(0,38)$. Nos estimations expliquent donc bien la probabilité de recours à une aide ménagère, mais parmi celles qui y ont eu recours au moins une heure dans la semaine, ce modèle ne rend pas bien compte des déterminants concernant le choix du volume d'heures. Les $R^{2}$ sont beaucoup plus faibles pour les modèles estimant la probabilité d'avoir recours à une aide extérieure pour le linge et la cuisine, et encore plus faibles pour ceux estimant le volume d'externalisation de ces deux tâches (autour de $5 \%$ de la variance totale dans les deux cas).

Les régressions logistiques indiquent que les coefficients sont très fortement positifs et significatifs pour ce qui est du recours à une aide ménagère, de la commande ou de la livraison de plats préparés, de l'utilisation du pressing et du recours à un système de garde rémunéré. Les régressions linéaires portant sur le volume des tâches montrent que, parmi les femmes appartenant à un ménage qui a recours à une forme d'externalisation, l'impact du salaire sur le volume de recours est faible mais significatif dans le cas des aides au ménage, du recours au pressing et des systèmes de garde d'enfants. Il est à l'inverse non significatif pour ce qui est de la proxy «commande et livraison de plats préparés », dans le cas de la cuisine. Pour cette tâche, le salaire explique la probabilité d'avoir recours à une aide extérieure, mais non le volume choisi par les couples, ce qui renforce selon nous avant tout le fait qu'il s'agit d'une approximation très partielle des substituts possibles pour cette tâche. Dans le cas du ménage, pour les femmes ayant recours à au moins une heure d'aide ménagère, une augmentation d'une heure par semaine de l'aide nécessite une augmentation de 714 euros de salaire. L'effet est donc faible pour cette tâche, d'autant qu'il faut rappeler qu'il s'agit dans ce cas de femmes situées dans le dernier décile de la distribution des revenus (cf. tableau 5), ce qui limite davantage la généralisabilité du mécanisme identifié. Pour le recours à une aide rémunérée de garde d'enfants, l'effet est plus fort puisqu'une augmentation d'une heure par semaine de ce service équivaut à une augmentation de salaire de 235 euros pour un niveau de salaire médian. On remarque qu'il existe une relation non linéaire significative uniquement dans le cas de la garde d'enfants. En particulier, pour le ménage et le 
linge, le niveau de salaire influence de manière linéaire à la fois la probabilité de recours à une aide ménagère extérieure, et le volume de recours à cette aide.

\section{La recours à une aide extérieure n'explique qu'une faible part de la relation entre le temps de travail domestique des femmes et leur salaire}

La question qui nous intéresse plus directement ici est celle qui porte sur la part de l'association négative et concave entre salaire et temps de travail domestique qu'on peut effectivement attribuer à un recours à une aide extérieure. Killewald (2011) et Sullivan et Gershuny (2013) suggèrent à cet effet une approche simple permettant de contourner les problèmes d'identification mentionnés précédemment. Killewald (2011) propose en particulier d'estimer les modèles précédents (5)-(7) en y incluant une variable mesurant la fréquence $\mathrm{du}$ recours à un service extérieur par le ménage, pour la tâche concernée. Nous dénommerons ces modèles «bis », ils sont reportés dans le tableau 4. À titre d'exemple, le modèle (7bis) inclut une variable supplémentaire par rapport au modèle (7) qui indique combien de fois le ménage a eu recours au pressing durant la semaine de l'enquête. Si les coefficients de régression estimés pour le salaire (linéaire et quadratique) changent significativement entre les deux estimations, cela signifie qu'une partie de l'association entre salaire et temps de travail domestique opère en réalité à travers le recours à un service extérieur équivalent. Cet écart permet de saisir si la corrélation imputée à une relation directe entre salaire et temps de travail domestique est due à un effet indirect de l'augmentation du recours à des services extérieurs, liée à des niveaux plus élevés de salaire. Cette approche ne nous permet pas de mesurer l'effet du salaire sur le recours à des services marchands, ni de mesurer l'effet de la fréquence de ce recours sur la quantité de travail domestique des femmes, mais elle permet de contourner cette relation triangulaire en évaluant si la forme de la courbe estimée est modifiée par l'introduction de la variable proxy de recours à un service marchands équivalent. Or, Killewald (2011) remarque dans son étude que l'association entre les deux est plus faible qu'on pourrait le supposer, le recours à des services de substitution n'étant que très faiblement associé à une baisse de la charge domestique dans le cas des femmes américaines : il est faible dans le cas du ménage et minime pour ce qui est de la cuisine. Ce résultat a été appuyé par les études récentes de Sullivan et Gershuny (2013) et Craig et Baxter (2014), et l'état actuel de la recherche suggère que la part de l'impact des ressources économiques sur la diminution du temps de travail des femmes qui est effectivement due à une externalisation des tâches domestiques est très faible.

L'estimation des modèles «bis» indique le même type de résultat: le recours à une aide extérieure pour le ménage et le recours au pressing sont corrélés négativement et significativement à la quantité de travail domestique effectuée par les femmes (6bis et 7 bis), un résultat déjà mis en évidence dans le cas australien par Bittman et al. (2004). Le fait de commander ou de se faire livrer des plats préparés n'a pas d'impact sur le temps que les femmes passent à faire la cuisine (5bis), ce qui confirme les résultats, bien qu'ils portent sur des mesures différentes, de Craig et Baxter (2014). Intéressons nous à présent aux différences entre les modèles (6), (7) et les versions (bis). Pour le ménage et le linge, l'introduction de la variable de recours à un service extérieur diminue la pente de la courbe estimée (terme linéaire), et diminue sa concavité (terme quadratique). Ces écarts entre les modèles sont très faibles, ce qui indique que la part de corrélation entre le salaire et le temps consacré à ces tâches qui est effectivement due à une externalisation des tâches est faible. Dans le modèle (6bis), la relation entre le salaire et le volume de temps consacré au ménage est moins négative et plus concave que dans le modèle (6). En d'autres termes, le recours à une aide ménagère rémunérée explique une partie de l'association négative entre salaire et temps de travail ménager, mais elle explique aussi une partie de la non-linéarité observée dans le modèle (6), bien que les effets mesurés soient faibles. On observe un phénomène similaire dans le cas du linge, l'introduction de la variable indiquant la fréquence de recours au pressing diminue l'association négative entre salaire et temps passé à s'occuper du linge, et elle diminue également sa concavité.

Il semble donc, en résumé, que l'effet de l'achat de services extérieurs de substitution sur le travail domestique des femmes soit relativement faible dans le cas des femmes françaises occupées à plein au sein de couples bi-actifs. Elle concerne une petite fraction de l'échantillon observé, et, bien qu'on identifie un effet du salaire des femmes à la fois sur la probabilité et sur le volume de recours à l'externalisation, ces effets ne semblent expliquer qu'une très faible 
partie de la relation entre le salaire et le temps de travail domestique des femmes ${ }^{52}$.

De plus, étant données les limites liées à la mesure et à l'identification mentionnées précédemment ${ }^{53}$, il convient de rester prudent quant aux résultats et à la possibilité d'en tirer des conclusion générales sur l'externalisation des tâches domestiques au sein des couples français. On tente dans ce qui suit de formuler cette question de l'externalisation ainsi que les problèmes qu'elle soulève, afin d'y répondre de la meilleure manière possible pour notre étude, étant données les mesures disponibles.

\section{Marge intensive et marge extensive : les modifications de la « qualité » de la production domestique}

L'augmentation des ressources économiques des femmes entraîne une diminution de la quantité de travail domestique qu'elles effectuent, et ce temps ne semble que partiellement compensé par une prise en charge du conjoint ou une externalisation sur le marché. La littérature récente (Killewald, 2011 ; Craig et Baxter, 2014) a nettement mis l'accent sur l'adaptation des attentes des femmes en termes de «qualité » de la production domestique. L'augmentation des revenus des femmes aurait comme conséquence une diminution globale de la production domestique et non uniquement de celle effectuée par les femmes ; certaines tâches ne seraient simplement plus considérées comme nécessaires à la vie du ménage. Cette hypothèse de "qualité " n'est pas toujours claire quant aux idées qu'elle cherche à introduire, et nous aimerions ici en clarifier le sens, quitte à en restreindre l'ambition. Selon notre interprétation a minima, l'hypothèse exprime différemment l'idée selon laquelle il n'y a aurait pas un volume fixé de tâches, au niveau du ménage, et dont il faudrait simplement décider du processus de production. L'idée sous-jacente, en particulier dans les modèles « relatifs », est que le ménage peut être identifié à une petite structure de production, qui fixerait son niveau de production en amont du reste des décisions ${ }^{54}$. Si l'on suit cette conception, le couple aurait à prendre deux types de décisions, d'une part il faudrait choisir entre une production en interne et une production en externe, d'autre part il s'agirait de négocier l'allocation du travail en interne, entre les deux conjoints. $\mathrm{La}$ présente étude contribue à montrer que ce qu'on observe est plus complexe: l'élasticité des tâches au salaire des femmes n'est pas constante selon le niveau de salaire, elle diffère entre les hommes et les femmes, et peut également différer par type de tâche. En d'autres termes, l'interprétation en termes de " qualité », dans sa version minimale, replace les femmes au centre de l'analyse et insiste sur le fait qu'elles peuvent simplement décider d'en "faire moins ». Pour reprendre une notion issue de l'analyse économique ${ }^{55}$, lorsque le salaire des femmes augmente, il y aurait des modifications de la marge « intensive » (externalisation sur le marché ou substitution de travail domestique supplémentaire des hommes) qui maintiendraient le même volume global de travail domestique au sein du couple, mais également des modifications de la marge " extensive », à savoir une adaptation par le volume de la production domestique. Nos résultats montrent par ailleurs que les modifications de la marge " extensive » sont différents selon la tâche considérée: le volume relatif des tâches effectuées peut en effet varier en fonction des niveaux de salaire. Notre modèle prédit en particulier, ce que montre la figure III, que pour des niveaux élevés de revenus, le poids du ménage diminue par rapport à celui de la cuisine, et le poids de ces deux « corvées » diminue lui aussi face à celui du child care.

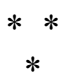

Nous avons pu montrer l'existence d'effets monotones négatifs, linéaire et non linéaire, du salaire des femmes sur les temps qu'elles consacrent aux différentes tâches domestiques. Nous avons également mis en avant un impact linéaire et bien plus faible du salaire des femmes sur le temps de travail domestique du conjoint. La diminution que les femmes parviennent ainsi à « acheter » transite avant tout par une diminution des tâches ménagères et du temps passé en cuisine. À l'inverse, le temps passé à s'occuper, éduquer et élever les enfants est insensible à ces modifications de revenus pour l'échantillon considéré ici. Notre étude se situe dans la lignée des critiques d'England (2011), Gupta (2007) et Sullivan (2011) qui insistent sur la nécessité

52. Le fait que le recours soit concentré sur une petite partie de la population peut être une des explications de la faiblesse des effets globaux du recours à l'externalisation des tâches.

53. Sur ces différents points, des solutions envisageables sont exposées en conclusion.

54. Les modèles économiques de production domestique sont en effet souvent séquentiels de ce point de vue, voir Gronau (1977). Et lorsque les équations sont couplées, le prix, donc en un sens la valeur ou la qualité $d u$ " bien domestique " produit est toujours connue et paramétrée à l'avance, voir en particulier Rapoport et al. (2011) et Thibout (2014).

55. Bien que l'emploi de ces deux notions soit différent en économie. 
de recentrer l'analyse sur le salaire des femmes, plutôt que de s'intéresser aux ressources relatives et au modalités de la négociation entre conjoints que celles-ci permettent d'influencer. Il semble en effet que l'enjeu central concerne avant tout la capacité des femmes à transformer ou non des acquis économiques en gain temporels; à déléguer aux hommes, ou au marché, certaines tâches laborieuses qui traditionnellement leur échoient davantage. Selon les mots de Delphy (2003, p. 66) : «La négociation ne marche pas ; les femmes partagent avec les hommes la notion que le temps des hommes est plus précieux, rapporte plus de valeur que le temps des femmes. [...] [L]e travail ménager n'est pas vu comme un vrai travail, mais comme quelque chose qui est sans valeur; il est vu comme quelque chose qui fait partie de la nature des femmes; qui fait partie de leurs obligations, parce que cela fait partie d'être une femme. Et être une femme, c'est bien le moins qu'on puisse exiger d'une femme. ».

La relation fortement négative et non linéaire que nous avons mise au jour dans le rapport entre le salaire des femmes et leur temps de travail domestique, à l'instar de Killewald et Gough (2010) et de Hook (2015), se retrouve au niveau d'une mesure globale des tâches et au niveau de postes spécifiques comme le ménage et le linge. À l'opposé, la cuisine évolue linéairement avec le salaire des femmes. La seconde partie de l'article visait à décrire plus en détail cette relation négative et non linéaire, en montrant que les mécanismes de substitution ne compensent que partiellement le temps de travail dont les femmes se défont. D'une part, l'effet sur le temps de travail domestique des hommes du salaire de la femme est linéaire et plus de 4 fois inférieur à l'effet sur le temps de la femme. D'autre part, l'effet d'un recours aux services extérieurs ne semble expliquer qu'une faible part de l'impact des ressources économiques sur le temps de travail domestique des femmes, bien que le pouvoir explicatif des modèles estimés à ce stade soit très faible. En particulier, on ne trouve pas de relation non linéaire symétrique dans le cas des hommes, et, étant donnée la faiblesse des résultats observés sur le recours à l'externalisation, il est à ce stade difficile d'expliquer la forme fonctionnelle identifiée par des mécanismes qui se joueraient au niveau de l'achat de services extérieurs au ménage. Nous pensons que la description précise des mécanismes menant à cette nonlinéarité constitue une tâche importante pour les recherches à venir.

D'un point de vue quantitatif, cela nécessite en particulier d'adopter une perspective longitudinale ${ }^{56}$ afin d'observer l'impact de modifications des revenus sur le temps de travail domestique effectué en interne, ou externalisé57. Nous avons également mis en avant la nécessité de se doter de mesures plus précises des substituts marchands aux tâches ménagères, ce qui implique de réfléchir à la fois à de meilleurs indicateurs ${ }^{58}$ et à un ensemble de variables supplémentaires permettant de raisonner à structure de production domestique équivalente. En particulier, prendre en compte la surface du logement pour ce qui est des aides ménagères ou le montant de dépenses en vêtements pour ce qui est du pressing permettrait de mieux comprendre la relation entre salaires, externalisation et temps de travail domestique des femmes. Pour la cuisine, il faudrait réfléchir plus précisément à la question portant sur les substituts possibles, en incluant par exemple le montant dépensé dans l'achat de plats préparés (hors commande ou livraison), le fait d'aller au restaurant ou d'aller manger dans la famille. Enfin, il s'agirait de s'interroger sur les effets possibles de la combinaison de plusieurs activités, ce qu'on nomme le multitasking, bien que Sullivan et Gershuny (2013) aient émis des doutes quant aux effets possibles de ce phénomène.

Le débat sur la répartition du travail domestique a été initié par un travail ethnographique effectué par Hochschild et Machung ; et depuis la publication de cet ouvrage, la question est devenue essentiellement quantitative (Sullivan, 2011 ; England, 2011). La publication des travaux de Lyonette et Crompton (2014) a néanmoins permis de rappeler le cadre et la complexité des questions ethnographiques de départ; et une étude similaire pour le cas de la France permettrait sans doute d'affiner considérablement les hypothèses qu'on cherche à tester. D'un point de vue qualitatif, en effet, un

56. Les études s'appuyant sur des données de panel présentent les mêmes types de conclusions, simplement il semble que les coefficients liés aux ressources économiques soient en général plus faibles dans ce cas, voir en particulier Killewald et Gough (2010), et Bertrand et al. (2015) pour un exemple de comparaison entre estimations en coupe et en panel.

57. Aucun modèle structurel complet n'existe à l'heure actuelle en microéconomie qui permettrait de tester des hypothèses précises en termes de substitution entre le temps de chaque conjoint et les alternatives externes. La thèse de Thibout (2014) constitue une nette avancée en la matière, elle y plaide dans son travail avec Sofer pour qu'un tel agenda de recherche se mette en place.

58. Deux solutions nous semblent envisageables à ce stade, la première serait de mesurer le niveau de dépenses consacré à chaque poste, afin d'avoir une idée plus précise du volume. La seconde serait d'inclure dans les carnets journaliers un ensemble de questions ayant trait aux tâches domestiques externalisées: pour l'ensemble des tâches les plus communément substituables, il s'agirait d'indiquer la présence de ces substituts dans les activités quotidiennes. 
certain nombre d'interrogations particulières portent sur ce niveau «incompressible» de tâches non-délaissées par les femmes : comment gèrent-elles un conflit entre les impératifs venant du travail marchand, et la volonté de s'impliquer temporellement et émotionnellement dans la vie domestique du ménage? Si cette adaptation des attentes existe, comment s'effectue-t-elle, par quelles tâches et est-ce formulé de la sorte par les femmes enquêtées? Nous ne sommes pas ici partis de critères a priori comme celui de «l'efficience », ou l'« inefficience» (Thibout, 2014) de la répartition du travail domestique au sein des couples, mais il est vrai que les choix effectués par les conjoints ne sont pas totalement séquentiels (au sens où le choix de la femme constituerait une première étape indépendante), ce que suppose notre approche. Ils sont effectués de manière dynamique, dans l'interaction et la pratique quotidienne des différentes tâches (West et Zimmerman 1987). Là encore, des études qualitatives précises pensant ces choix dans leur exhaustivité et leurs entrecroisements permettrait d'améliorer notre connaissance du type de déterminant dont on cherche à évaluer l'impact. De ce point de vue, nous avons largement remis en cause l'existence d'un effet quantitatif de neutralisation de la déviance de genre, mais cela ne veut pas dire que nous mettons en doute l'existence de comportements symboliques forts observables à un niveau empirique plus fin. La notion de « performance » était chez Goffman avant tout mobilisée pour comprendre les comportements individuels, construits dans les interactions quotidiennes, et il apparaît essentiel de comprendre le sens qu'attribuent aux différentes tâches les époux qui les effectuent. Comment les conjoints réagissent-ils aux modifications de leur statut économique respectif? Quelles tâches sont identifiées comme des corvées et comment évolue le temps qu'y consacrent les femmes à mesure que leurs revenus changent? Lors, en particulier, de trajectoires professionnelles ascendantes ou descendantes. Seule l'étude longitudinale des parcours de vie permettra de comprendre le détail de ces comportements sociaux, et en retour de formuler des hypothèses quantitatives plus fines quant à la description du rapport entre ressources économiques des femmes et inégalités dans la gestion des charges familiales.

Cet agenda prend acte des critiques émises par Sullivan (2011) et England (2011) à l'égard de la littérature quantitative sur le sujet, qui a eu tendance à interpréter rapidement les effets non linéaires des ressources relatives en termes de comportements sociaux très généraux (Akerlof et Kranton, 2000 ; Bertrand et al., 2015), et nous laissons ici ouverte l'interprétation possible des logiques causales pouvant expliquer cette forme fonctionnelle non linéaire dans le cas du salaire des femmes. Nous émettons simplement la double hypothèse que, d'une part, la contrainte liée à la nécessité de maintenir un niveau minimal, incompressible de tâches peut jouer, et d'autre part que cette incompressibilité, cette inélasticité croissante du temps domestique à mesure que les revenus augmentent, peut être au moins en partie due au poids de socialisations genrées différentielles entre hommes et femmes qui se perpétuent. Celles-ci mettent en avant des responsabilités domestiques qui sont encore vues et ressenties comme le devoir des femmes, et qui peuvent limiter le désir qu'ont les femmes salariées de se délester complètement des charges familiales, même dans des situations où les contraintes liées au travail marchand rendent cette articulation matériellement difficile (Acker, 2009) et psychologiquement coûteuse (Hochschild et Machung, 1989 ; Bereni et al., 2011).

\section{BIBLIOGRAPHIE}

Acker J. (1988), « Class, gender, and the relations of distribution », Signs, n ${ }^{\circ}$, vol. 3 pp. 473-497.

Acker J. (2009), « From glass ceiling to inequality regimes », Sociologie du travail, $\mathrm{n}^{\circ} 51$, vol. 2 , pp. 199-217.

Akerlof G.A. et Kranton R.E. (2000), « Economics and identity», The Quarterly Journal of Economics, $\mathrm{n}^{\circ} 115$, vol. 3, pp. 715-753.
Apps P.F. et Rees R. (1997), «Collective labor supply and household production », The Journal of Political Economy, $\mathrm{n}^{\circ}$ 105, vol. 1, pp. 178-190.

Baizán P., Dominguez M. et González M.J. (2014), "Couple bargaining or socio-economic status? why some parents spend more time with their children than others », European Societies, $\mathrm{n}^{\circ}$ 16, vol. 1, pp. 3-27. 
Becker G.S. (1985), « Human capital, effort, and the sexual division of labor », Journal of Labor Economics, pp. S33-S58.

Becker G.S. (1965), «A theory of the allocation of time ", The Economic Journal, n 299 , vol. 75, pp. 493-517.

Becker G.S. (2009), A Treatise on the Family, Harvard University Press, Cambridge.

Bereni L., Marry C., Pochic S. et Revillard A. (2001), « Le plafond de verre dans les ministères: regards croisés de la sociologie du travail et de la science politique », Politiques et management public, $\mathrm{n}^{\circ} 28$, vol. 2 .

Bertrand M., Kamenica E. et Pan J. (2015), «Gender identity and relative income within households ", The Quarterly Journal of Economics, $\mathrm{n}^{\circ} 130$, vol. 2, pp. 571-614.

Bessière C. (2004), « Vaut mieux qu'elle travaille à l'extérieur ! », Cahiers du genre, n 2, pp. 93-114.

Bessière C. (2008), « Le soi, le couple et la maisonnée exploitante : l'argent dans les couples mixtes agriculteur/salariée en France ", dans L'usage de l'argent dans le couple: pratiques et perceptions des comptes amoureux: perspective internationale, chapitre 8, Éditions L'Harmattan, Paris.

Bianchi S.M., Milkie M.A., Sayer L.C. et Robinson J.P. (2000), « Is anyone doing the housework? trends in the gender division of household labor », Social Forces, $\mathrm{n}^{\circ}$ 79, vol. 1, pp. 191-228.

Bittman M., Rice J.M. et Wajcman J. (2004), «Appliances and their impact: the ownership of domestic technology and time spent on household work », The British Journal of Sociology, $\mathrm{n}^{\circ} 55$, vol. 3, pp. 401-423.

Bittman M., England P., Sayer L., Folbre N., et Matheson G. (2003), « When does gender trump money? Bargaining and time in household work », American Journal of Sociology, $\mathrm{n}^{\circ} 109$, vol. 1, pp. 186-214.

Bloemen H. et Stancanelli E. (2008), « How do spouses allocate time: the impact of wages and income », IZA , Institute for the Study of Labor, Discussion Papers, ${ }^{\circ}$ 3679, vol. 7, Bonn.

Brines J. (1994), « Economic dependency, gender, and the division of labor at home ", American Journal of Sociology, $\mathrm{n}^{\circ}$ 100, vol. 3 , pp. 652-688.

Brousse C. (1999), « La répartition du travail domestique entre conjoints reste très largement spécialisée et inégale », France portrait social 2000, Insee, pp. 137-151.

Chiappori P.A., Fortin B. et Lacroix G. (2002), " Marriage market, divorce legislation, and household labor supply », Journal of Political Economy, $n^{\circ} 110$, vol. 1, pp. 37-72.

Chiappori P.A. (1992), «Collective labor supply and welfare », Journal of Political Economy, $\mathrm{n}^{\circ} 100$, vol. 3, pp. 437-467.

Chiappori P.A. (1997), « Introducing household production in collective models of labor supply $\gg$, Journal of Political Economy, n ${ }^{\circ}$ 105, vol. 1, pp. 191-209.

Coltrane S. (2000), «Research on household labor: Modeling and measuring the social embeddedness of routine family work », Journal of Marriage and Family, n ${ }^{\circ}$ 62, vol. 4, pp. 208-1233.

Connelly R. et Kimmel J. (2009), «Spousal influences on parents and non-market time choices "), Review of Economics of the Household, $\mathrm{n}^{\circ}$ 7, vol. 4, pp. 361-394.

Courduriès J. (2008), « L'argent chez les couples gays ", dans L'usage de l'argent dans le couple: pratiques et perceptions des comptes amoureux: perspective internationale, chapitre 7, pp. 259-295.

Craig L. et Baxter J. (2014), « Domestic outsourcing, housework shares and subjective time pressure : Gender differences in the correlates of hiring help », Social Indicators Research, pp. 1-18.

Delphy C. (2003), «Par où attaquer le partage inégal du travail ménager? », Nouvelles questions féministes, $\mathrm{n}^{\circ} 22$, vol.3, pp. 47-71.

Delphy C. et Leonard D. (1992), Familiar exploitation: A new analysis of marriage in contemporary western societies, Polity Press, Cambridge.

Delphy C. (2001), L'ennemi principal, tome 2 : L'économie politique du patriarcat, Editions Syllepse, Paris.

De Singly F. (1987), Fortune et infortune de la femme mariée, PUF, Paris.

Domnguez-Folgueras M. (2013), « Is cohabitation more egalitarian? the division of household labor in five european countries », Journal of Family Issues, n 34, vol. 12, pp. 1623-1646.

England P. et Folbre N. (2005), " Gender and economic sociology ", dans The Handbook of Economic Sociology 2, pp. 627-649, Princeton University Press, Princeton. 
England P. (2011), « Missing the big picture and making much ado about almost nothing: Recent scholarship on gender and household work $"$, Journal of Family Theory \& Review, ${ }^{\circ} 3$, vol. 1, pp. 23-26.

Evertsson M. et Nermo M. (2007), «Changing resources and the division of housework: A longitudinal study of swedish couples », European Sociological Review, $\mathrm{n}^{\circ} 23$, vol. 4, pp. 455-470.

Gershuny J., Bittman M. et Brice J. (2005), «Exit, voice, and suffering: Do couples adapt to changing employment patterns? ", Journal of Marriage and Family, n ${ }^{\circ}$ 67, vol. 3, pp. 656-665.

Gershuny J. (2003), Changing times: Work and leisure in postindustrial society, Oxford University Press, Oxford.

Goffman E. (1979), Gender advertisements, vol. 3, Macmillan, Londres.

Greenstein T.N. (2000), « Economic dependence, gender, and the division of labor in the home: A replication and extension ", Journal of Marriage and Family, $\mathrm{n}^{\circ} 62$, vol. 2, pp. 322-335.

Gronau R. (1977), « Leisure, home production, and work - the theory of the allocation of time revisited », Journal of Political Economy, n ${ }^{\circ} 85$, vol. 6, pp. 1099-1123.

Gupta S. et Ash M. (2008), «Whose money, whose time? a nonparametric approach to modeling time spent on housework in the united states », Feminist Economics, n ${ }^{\circ} 14$, vol. 1, pp. 93-120.

Gupta S. (2006), « Her money, her time: Women's earnings and their housework hours », Social Science Research, $\mathrm{n}^{\circ}$ 35, vol. 4, pp. 975-999.

Gupta S. (1999), «The effects of transitions in marital status on men's performance of housework », Journal of Marriage and the Family, $\mathrm{n}^{\circ}$ 61, vol. 3, pp. 700-711.

Gupta S. (2007), « Autonomy, dependence, or display? the relationship between married women's earnings and housework », Journal of Marriage and Family, ${ }^{\circ} 69$, vol. 2, pp. 399-417.

Guryan J., Hurst E. et Kearney M. (2008), « Parental education and parental time with children », Journal of Economic Perspectives, $\mathrm{n}^{\circ} 22$, vol. 3, pp. 23-46.

Russell Hill C. et Stafford F.P. (1980), « Parental care of children: Time diary estimates of quantity, predictability, and variety », Journal of Human Resources, $\mathrm{n}^{\circ} 15$, vol. 2, pp. 219-239.

Hirschman A.O. (1970), Exit, voice, and loyalty: Responses to decline in firms, organizations, and states, Harvard University Press, Cambridge.

Hochschild A.R. et Machung A. (1989), The second shift, Avon Books, New York.

Hook J.L. et Wolfe C.M. (2013), « Parental involvement and work schedules: Time with children in the united states, germany, norway and the united kingdom », European Sociological Review, n 29 , vol. 3, pp. 411-425.

Hook J.L. (2010), "Gender inequality in the welfare state: Sex segregation in housework, 1965-2003 », American Journal of Sociology, $\mathrm{n}^{\circ} 115$, vol. 5, pp. 1480-1523.

Hook J.L. (2015), « Women housework and quadratic associations: An end to gender deviance neutralization? "), Paper to be presented at the 2015 Population Association of America Annual Meeting, Princeton University.

Horrell S. et Humphries J. (1995), « Women's labour force participation and the transition to the male-breadwinner family, 1790-1865 », The Economic History Review, n ${ }^{\circ} 48$, vol. 1, pp. 89-117.

Horrell S. et Humphries J. (1996), « The origins and expansion of the male breadwinner family: the case of nineteenth-century Britain », International Review of Social History, $n^{\circ} 42$, vol. S5, pp. 25-64.

Killewald A. et Gough M. (2010), «Money isn't everything: Wives' earnings and housework time », Social Science Research, n³9, vol. 6, pp. 987-1003.

Killewald A. (2011), « Opting out and buying out: Wives' earnings and housework time », Journal of Marriage and Family, ${ }^{\circ} 73$, vol. 2, pp. 459-471.

Lundberg S. et Pollak R.A. (1993), « Separate spheres bargaining and the marriage market», Journal of Political Economy, $\mathrm{n}^{\circ}$ 101, vol; 6, pp. $988-1010$.

Lundberg S. et Pollak R.A. (1994), « Noncooperative bargaining models of marriage $»$, American Economic Review, n 84, vol. 2, pp. 132-137.

Lyonette C. et Crompton R. (2014), « Sharing the load? partners' relative earnings and the division of domestic labour », Work, Employment \& Society, $\mathrm{n}^{\circ} 29$, vol. 1, pp. 23-40. 
Manser M. et Brown M. (1980), « Marriage and household decision-making: A bargaining analysis ", International Economic Review, $\mathrm{n}^{\circ} 21$, vol. 1, pp. 31-44.

Marini M.M. et Shelton B.A. (1993), « Measuring household work: Recent experience in the united states ", Social Science Research, $\mathrm{n}^{\circ} 22$, vol. 4, pp. 361-382.

McElroy M.B. et Horney M.J. (1981), « Nashbargained household decisions: Toward a generalization of the theory of demand », International Economic Review, n ${ }^{\circ} 2$, vol. 2, pp. 333-349.

Niemi I. (1993), « Systematic error in behavioural measurement: Comparing results from interview and time budget studies $»$, Social Indicators Research, $\mathrm{n}^{\circ} 30$, vol. 2-3, pp. 229-244.

Ollier-Malaterre A. (2008), «Comment les employeurs appréhendent-ils la vie hors travail de leurs salariés? [une comparaison entre le modèle français et le modèle anglo-américain "), Recherches et prévisions, $\mathrm{n}^{\circ}$ 92, vol. 1, pp. 47-60.

Pailhe A. et Solaz A. (2008), « Time with children: Do fathers and mothers replace each other when one parent is unemployed? ", European Journal of Population/Revue Européenne de Démographie, ${ }^{\circ} 24$, vol. 2, pp. 211-236.

Pallié B. (1993), «Estimating the volume of unpaid activities in canada, 1992: An evaluation of data from the general social survey », General Social Survey, Working Paper n 10.

Ponthieux S. et Schreiber A. (2006), «Dans les couples de salariés, la répartition du travail domestique reste inégale », Données Sociales, La Société Française, Paris.

Ponthieux S. et Meurs D. (2015), « Gender Equality », dans Atkinson-Bourguignon, Handbook of Income Distribution, Vol. 2.B., Elsevier, Oxford.

Presser H.B. (1994), «Employment schedules among dual-earner spouses and the division of household labor by gender », American Sociological Review, ${ }^{\circ} 21$, vol. 1, pp. 348-364.

Rapoport B., Sofer C. et Solaz A. (2011), «Household production in a collective model: some new results », Journal of Population Economics, $\mathrm{n}^{\circ} 24$, vol. $1, \mathrm{n}^{\circ} 59$, vol. 3, pp. 23-45.

Régnier-Loilier A. (2009), «L'arrivée d'un enfant modifie-t-elle la répartition des tâches domestiques au sein du couple? ", Population et Sociétés, $\mathrm{n}^{\circ}$ 461, pp. 1-4.
Rizavi S.S. et Sofer C. (2008), « The division of labour within the household: Is there any escape from traditional gender roles », Technical Report, Working Paper.

Ross C.E. (1987), " The division of labor at home », Social Forces, n ${ }^{\circ}$, vol. 3, pp. 816-833.

Roy D. (2011), «La contribution du travail domestique au bien-être matériel des ménages : une quantification à partir de l'enquête emploi du temps », Insee, Direction des études démographiques et sociales, Document de travail ${ }^{\circ}$ F1104.

Schneider D. (2012), « Gender deviance and household work: The role of occupation », American Journal of Sociology, ${ }^{\circ} 117$, vol. 4, pp. 1029-1072.

Solaz A. (2005), « Division of domestic work: Is there adjustment between partners when one is unemployed? ", Review of Economics of the Household, $\mathrm{n}^{\circ} 3$, vol. 4, pp. 387-413.

Solberg E.J. et Wong D.C. (1992), « Family time use: leisure, home production, market work, and work related travel », Journal of Human Resources, $\mathrm{n}^{\circ} 27$, vol. 3, pp. 485-510.

Stewart J. (2013), « Tobit or not tobit?», Journal of Economic and Social Measurement, $\mathrm{n}^{\circ} 38$, vol. 3, pp. 263-290.

Sullivan O. et Gershuny J. (2013), « Domestic outsourcing and multitasking: how much do they really contribute? ", Social Science Research, $\mathrm{n}^{\circ} 42$, vol. 5, pp. 1311-1324.

Sullivan O. (2011), «An end to gender display through the performance of housework? A review and reassessment of the quantitative literature using insights from the qualitative literature », Journal of Family Theory \& Review, n ${ }^{\circ}$, vol. 1, pp. 1-13.

Sullivan O. (2013), «What do we learn about gender by analyzing housework separately from child care? some considerations from time-use evidence », Journal of Family Theory \& Review, $\mathrm{n}^{\circ} 5$, vol. 2, pp. 72-84.

Thibout C. (2014), Efficience et stéréotypes de genre : applications à l'allocation des ressources dans les ménages et aux choix éducatifs, thèse de doctorat, Université Paris 1 - Panthéon Sorbonne.

Townsley E. (1998), « Wives'and husbands'housework reporting gender, class, and social desirability », Gender \& Society, n 12, vol. 2, pp. 188-218.

West, C. et Zimmerman D. (1987), « Doing gender », Gender \& Society, n 1, vol. 2, pp. 125-151. 


\section{DONNÉES POUR LES JOURS DE WEEK-END}

Le tableau ci-dessous présente des statistiques descriptives ayant pour objectif de contrôler la spécificité éventuelle du travail domestique les jours de week-end. Les ménages effectuent en moyenne 30 minutes de travail domestique de plus que les autres jours (282 minutes contre 252 minutes) et la répartition du volume global y est également plus égalitaire : les hommes et les femmes consacrant chacun 15 minutes de plus aux tâches domestiques, on observe une réduction de l'écart dans la répartition des tâches de 25 points de pourcentage. Lorsqu'on éclate le volume global en groupes de tâches spécifiques, la répartition des tâches s'avère très sensiblement la même pour les postes cuisine, ménage, linge et «s'occuper des enfants ». Les différences avec les jours de semaines sont les moins marquées pour les tâches que nous pourrions imaginer être effectuées en priorité le week-end: le linge et le ménage. Les différences en termes de volume global que nous observons sont avant tout dues à deux tâches particulières: les tâches administratives comme la " gestion du ménage ", " faire ses comptes ", " courrier administratif » et les " transports non-professionnels "; tâches qui, dans nos données, sont plus égalitaires que les quatre postes centraux analysés en détail dans la section 6 . Le recours aux services extérieurs est également très proche de ce qu'on observe pour les jours de semaine, à l'exception du recours à un système de garde rémunéré, qui diminue de 5 minutes (16\%) le week end.

Tableau

\section{Travail domestique le week-end}

\begin{tabular}{|l|c|c|c|}
\hline \multicolumn{1}{|c|}{ Variable } & Femmes (834) & Hommes (834) & $\begin{array}{c}\text { Différence } \\
\text { (Pourcentage) }\end{array}$ \\
\hline Temps quotidien alloué au travail domestique (minutes) & $189,93(4,64)$ & $92,35(3,47)$ & 105,7 \\
Volume global & $49,12(1,7)$ & $21,66(1,21)$ & 126,8 \\
Faire la cuisine & $63,41(2,47)$ & $25,16(1,58)$ & 152,8 \\
Faire le ménage & $21,88(1,36)$ & $1,73(0,39)$ & 1164,7 \\
S'occuper du linge & $40,31(2,69)$ & $18,73(1,65)$ & 115,2 \\
S'occuper des enfants & 0,69 & 0,31 \\
Répartition du travail domestique, niveau agrégé & 37,30 & 124,9 \\
\hline Le ménage a eu recours à des services extérieurs (en pourcentages) & 12,27 & - \\
Pressing & 21,21 & - \\
Aide rémunérée pour le ménage & 25,40 & - \\
Commande et livraison de plats préparés & & - \\
Système de garde rémunérée des enfants & & - \\
\hline
\end{tabular}

Lecture: les écarts-types sont fournis quand cela est nécessaire. La part agrégée est calculée sur la totalité de l'échantillon alors que les parts individuelles sont calculées par ménage. Nous ne considérons que les couples en cohabitation, ainsi le nombre d'enfants est le même pour les hommes et les femmes. De la même manière, le recours aux services extérieurs indique le pourcentage de ménages ayant indiqué avoir eu recours à ce types de solution au moins une fois durant la semaine de l'enquête.

Champ : couples hétérosexuels bi-actifs occupés, exerçant une activité salariée à temps complet, en situation de cohabitation. Source : enquête Emploi du Temps 2009-2010, Insee. 
Tableau

Régressions logistiques et linéaires pour le recours à l'externalisation

\begin{tabular}{|c|c|c|c|c|c|c|c|c|}
\hline \multirow{2}{*}{ Modèle } & \multicolumn{4}{|c|}{ Régressions logistique, probabilité de recours } & \multicolumn{4}{|c|}{ Régressions linéaires,volume de recours } \\
\hline & Ménage & Linge & Cuisine & Enfants & Ménage & Linge & Cuisine & Enfants \\
\hline $\begin{array}{l}\text { Variable } \\
\text { dépendante }\end{array}$ & $\begin{array}{c}\text { Aide } \\
\text { Ménagère } \\
\text { rémunérée }\end{array}$ & $\begin{array}{l}\text { Recours } \\
\text { au pressing }\end{array}$ & $\begin{array}{l}\text { Commande } \\
\text { livraisons } \\
\text { de plats }\end{array}$ & $\begin{array}{l}\text { Garde } \\
\text { rémunérée }\end{array}$ & Heures & Fréquence & Fréquence & Heures \\
\hline Salaire femme & $\begin{array}{c}0,0013^{\star \star \star} \\
(0,00)\end{array}$ & $\begin{array}{c}0,00037^{\star *} \\
(0,00)\end{array}$ & $\begin{array}{c}0,00085^{\star \star \star} \\
(0,00)\end{array}$ & $\begin{array}{c}0,00063^{\star \star} \\
(0,00)\end{array}$ & $\begin{array}{c}0,0014^{*} \\
(0,00)\end{array}$ & $\begin{array}{c}0,00085^{\star \star} \\
(0,00)\end{array}$ & $\begin{array}{c}0,00038 \\
(0,00)\end{array}$ & $\begin{array}{c}0,00557^{\star \star} \\
(0,00)\end{array}$ \\
\hline Salaire femme ${ }^{2}$ & $\begin{array}{c}-8,68 \mathrm{e}-08 \\
(0,00)\end{array}$ & $\begin{array}{c}-2,52 \mathrm{e}-08 \\
(0,00)\end{array}$ & $\begin{array}{c}-1,30 \mathrm{e}-07 \\
(0,00)\end{array}$ & $\begin{array}{c}-4,42 \mathrm{e}-08 \\
(0,00)\end{array}$ & $\begin{array}{c}-0,00000014 \\
(0,00)\end{array}$ & $\begin{array}{c}-0,00000016 \\
(0,00)\end{array}$ & $\begin{array}{c}-0,00000020 \\
(0,00)\end{array}$ & $\begin{array}{c}-0,000000879^{\star \star} \\
(0,00)\end{array}$ \\
\hline Salaire conjoint & $\begin{array}{c}0,00031^{\star \star *} \\
(0,00)\end{array}$ & $\begin{array}{c}0,00039^{\star \star \star} \\
(0,00)\end{array}$ & $\begin{array}{c}0,00013^{\star \star \star} \\
(0,00)\end{array}$ & $\begin{array}{c}-0,000087 \\
(0,00)\end{array}$ & $\begin{array}{c}0,000105 \\
(0,00)\end{array}$ & $\begin{array}{c}0,00018^{\star *} \\
(0,00)\end{array}$ & $\begin{array}{c}0,00020 \\
(0,00)\end{array}$ & $\begin{array}{c}0,0000419 \\
(0,00)\end{array}$ \\
\hline Âge & $\begin{array}{l}0,38^{\star \star \star} \\
(0,13)\end{array}$ & $\begin{array}{l}-0,034 \\
(0,056)\end{array}$ & $\begin{array}{c}-0,0023 \\
(0,065)\end{array}$ & $\begin{array}{c}0,46^{\star \star \star} \\
(0,12)\end{array}$ & $\begin{array}{l}0,047 \\
(0,43)\end{array}$ & $\begin{array}{l}0,056 \\
(0,13)\end{array}$ & $\begin{array}{l}0,016 \\
(0,17)\end{array}$ & $\begin{array}{l}-1,58 \\
(1,26)\end{array}$ \\
\hline$\hat{A} \mathrm{e}^{2}$ & $\begin{array}{c}-0,0038^{\star \star \star} \\
(0,00)\end{array}$ & $\begin{array}{c}0,00065 \\
(0,00)\end{array}$ & $\begin{array}{c}-0,00071 \\
(0,00)\end{array}$ & $\begin{array}{c}-0,0067^{\star \star \star} \\
(0,02)\end{array}$ & $\begin{array}{l}2,57^{\star \star} \\
(0,00)\end{array}$ & $\begin{array}{c}-0,000082 \\
(0,00)\end{array}$ & $\begin{array}{c}-0,00035 \\
(0,00)\end{array}$ & $\begin{array}{c}0,0160 \text { * } \\
(0,02)\end{array}$ \\
\hline \multicolumn{9}{|l|}{ Enfant : } \\
\hline Âge $<3$ & $\begin{array}{l}0,48 \\
(0,29)\end{array}$ & $\begin{array}{l}0,33 \\
(0,17)\end{array}$ & $\begin{array}{r}-0,022 \\
(0,189)\end{array}$ & $\begin{array}{r}-0,44 \\
(0,23)\end{array}$ & $\begin{array}{l}-0,17 \\
(0,87)\end{array}$ & $\begin{array}{r}-0,44 \\
(0,41)\end{array}$ & $\begin{array}{r}-0,56 \\
(0,45)\end{array}$ & $\begin{array}{c}16,94^{\star \star \star} \\
(1,55)\end{array}$ \\
\hline $3 \leq$ âge $<6$ & $\begin{array}{l}0,50^{\star \star} \\
(0,21)\end{array}$ & $\begin{array}{l}0,14 \\
(0,14)\end{array}$ & $\begin{array}{l}-0,14 \\
(0,16)\end{array}$ & $\begin{array}{l}1,41^{\star \star \star} \\
(0,17)\end{array}$ & $\begin{array}{l}-1,07^{\star} \\
(0,62)\end{array}$ & $\begin{array}{l}-0,11 \\
(0,34)\end{array}$ & $\begin{array}{l}-0,28 \\
(0,39)\end{array}$ & $\begin{array}{l}3,90^{\star \star \star} \\
(1,25)\end{array}$ \\
\hline Âge $<18$ & $\begin{array}{l}0,20 \\
(0,11)\end{array}$ & $\begin{array}{c}-0,33^{\star \star \star} \\
(0,074)\end{array}$ & $\begin{array}{c}-0,16^{\star \star} \\
(0,09)\end{array}$ & $\begin{array}{l}0,42^{\star \star \star} \\
(0,096)\end{array}$ & $\begin{array}{l}0,98^{\star \star} \\
(0,39)\end{array}$ & $\begin{array}{l}0,25 \\
(0,17)\end{array}$ & $\begin{array}{l}0,18 \\
(0,22)\end{array}$ & $\begin{array}{l}-0,69 \\
(0,98)\end{array}$ \\
\hline \multicolumn{9}{|l|}{ Diplôme: } \\
\hline BEPC & $\begin{array}{l}0,58 \\
(0,45)\end{array}$ & $\begin{array}{r}-0,16 \\
(0,23)\end{array}$ & $\begin{array}{l}0,096 \\
(0,28)\end{array}$ & $\begin{array}{r}-0,62 \\
(0,48)\end{array}$ & $\begin{array}{c}-0,697 \\
(1,51)\end{array}$ & $\begin{array}{l}0,845 \\
(0,55)\end{array}$ & $\begin{array}{l}0,234 \\
(0,71)\end{array}$ & $\begin{array}{l}4,483 \\
(4,88)\end{array}$ \\
\hline Baccalauréat & $\begin{array}{l}0,65 \\
(0,66)\end{array}$ & $\begin{array}{l}0,32 \\
(0,23)\end{array}$ & $\begin{array}{c}0,17 \\
(0,28)\end{array}$ & $\begin{array}{c}-0,039 \\
(0,36)\end{array}$ & $\begin{array}{c}-5,63^{\star *} \\
(2,43)\end{array}$ & $\begin{array}{l}1,28^{\star \star} \\
(0,57)\end{array}$ & $\begin{array}{l}0,38 \\
(0,73)\end{array}$ & $\begin{array}{l}2,77 \\
(3,56)\end{array}$ \\
\hline Licence & $\begin{array}{l}1,28 \\
(0,68)\end{array}$ & $\begin{array}{c}0,38 \\
(0,26)\end{array}$ & $\begin{array}{r}-0,12 \\
(0,32)\end{array}$ & $\begin{array}{l}0,30 \\
(0,38)\end{array}$ & $\begin{array}{c}-6,91^{\star \star \star} \\
(2,54)\end{array}$ & $\begin{array}{l}1,02 \\
(0,64)\end{array}$ & $\begin{array}{l}0,33 \\
(0,80)\end{array}$ & $\begin{array}{l}6,99^{*} \\
(3,64)\end{array}$ \\
\hline Master & $\begin{array}{l}1,57^{\star *} \\
(0,67)\end{array}$ & $\begin{array}{l}0,51 \\
(0,26)\end{array}$ & $\begin{array}{l}-0,12 \\
(0,31)\end{array}$ & $\begin{array}{l}0,35 \\
(0,38)\end{array}$ & $\begin{array}{c}-6,10^{\star *} \\
(2,52)\end{array}$ & $\begin{array}{l}0,74 \\
(0,63)\end{array}$ & $\begin{array}{l}0,47 \\
(0,80)\end{array}$ & $\begin{array}{l}2,17 \\
(3,60)\end{array}$ \\
\hline \multicolumn{9}{|l|}{ Zonage urbain : } \\
\hline Moyen pôle & $\begin{array}{l}0,025 \\
(0,47)\end{array}$ & $\begin{array}{c}-0,0058 \\
(0,26)\end{array}$ & $\begin{array}{c}0,72 \\
(0,43)\end{array}$ & $\begin{array}{c}0,19 \\
(0,35)\end{array}$ & $\begin{array}{c}0,77 \\
(1,57)\end{array}$ & $\begin{array}{l}0,28 \\
(0,65)\end{array}$ & $\begin{array}{l}1,98^{*} \\
(1,18)\end{array}$ & $\begin{array}{r}-3,25 \\
(3,04)\end{array}$ \\
\hline Grand pôle & $\begin{array}{c}-0,053 \\
(0,35)\end{array}$ & $\begin{array}{l}0,25 \\
(0,19)\end{array}$ & $\begin{array}{l}1,64^{\star \star \star} \\
(0,34)\end{array}$ & $\begin{array}{l}-0,14 \\
(0,25)\end{array}$ & $\begin{array}{l}1,152 \\
(1,13)\end{array}$ & $\begin{array}{l}0,466 \\
(0,46)\end{array}$ & $\begin{array}{c}-0,0876 \\
(0,94)\end{array}$ & $\begin{array}{c}-2,628 \\
(2,22)\end{array}$ \\
\hline Constante & $\begin{array}{c}-15,40^{\star \star \star} \\
(2,75)\end{array}$ & $\begin{array}{c}-1,90 \\
(1,08523)\end{array}$ & $\begin{array}{c}-2,56^{\star \star} \\
(1,261725)\end{array}$ & $\begin{array}{l}-10,75^{\star \star \star} \\
(2,206145)\end{array}$ & $\begin{array}{l}3,28 \\
(8,84)\end{array}$ & $\begin{array}{l}-1,56 \\
(2,69)\end{array}$ & $\begin{array}{l}1,18 \\
(3,16)\end{array}$ & $\begin{array}{l}42,60^{*} \\
(22,84)\end{array}$ \\
\hline$R^{2}$ & 0,2448 & 0,0856 & 0,0750 & 0,3753 & 0,119 & 0,049 & 0,056 & 0,380 \\
\hline$N$ & 1674 & 1674 & 1674 & 1674 & 190 & 630 & 368 & 393 \\
\hline
\end{tabular}

Lecture : ${ }^{* *}$ indique que le coefficient est significatif à $1 \%,{ }^{* *}$ à $5 \%$ et * à $10 \%$. On donne l'estimation des coefficients de régression pour chaque variable, avec entre parenthèses l'écart-type corrigé de l'hétéroscédasticité au niveau du cluster du ménage, Nous indiquons la significativité de ces coefficients à l'aide d'un test de Student, celle-ci est à interpréter à l'aide de la grille ci-dessus portant sur la p-value. L'interprétation de l'effet des variables indépendantes sur le temps de travail domestique se fait toutes choses égales par ailleurs : dans le premier modèle (deuxième colonne), 100 euros supplémentaires de salaire pour les femmes entraînent une augmentation de la probabilité pour le ménage d'avoir recours à une aide ménagère, par rapport à la probablité de ne pas y avoir recours, de 0.13, en contrôlant pour l'effet de l'ensemble des variables indiquées dans la première colonne.

Champ : couples hétérosexuels bi-actifs occupés, exerçant une activité salariée à temps complet, en situation de cohabitation. Source : enquête Emploi du Temps 2009-2010, Insee. 